\title{
ABSTRACT
}

Autophagy is an evolutionary conserved intracellular catabolic process of vital importance to cell and tissue homeostasis. Autophagy is implicated in the pathogenesis of atherosclerosis but participating cells, molecular mechanisms and functional outcomes have not been fully elucidated. T-cadherin, an atypical glycosylphosphatidylinositol-anchored member of the cadherin superfamily of adhesion molecules, is upregulated on smooth muscle cells (SMCs) ${ }^{1}$ in atherosclerotic lesions. Here, using rat and murine aortic SMCs as experimental models, we surveyed the ability of T-cadherin to regulate autophagy in SMCs during serum-starvation stress. Ectopic upregulation of T-cadherin in SMCs resulted in augmented autophagy characterized by increased autophagic flux, LC3-II abundance and autophagosome formation. Analysis of signal transduction pathway effectors and use of specific pharmacological inhibitors demonstrated that T-cadherin-associated enhancement of the autophagic response to serum-deprivation was dependent on MEK1/2/Erk1/2 activation and independent of $\mathrm{PI} 3 \mathrm{~K} / \mathrm{Akt} / \mathrm{mTORC} 1$, reactive oxygen species or endoplasmic reticulum stress. T-cadherin upregulation on SMCs conferred a survival advantage during prolonged serumstarvation which was sensitive to inhibition of MEK1/2/Erk1/2 by PD98059 or UO126 and to blockade of autophagy by chloroquine. Loss of T-cadherin expression in SMCs diminished autophagy responsiveness and compromised survival under conditions of serum-starvation. Overall our findings have identified T-cadherin as a novel positive regulator of autophagy and survival in SMCs. 


\section{T-cadherin promotes autophagy and survival in vascular smooth muscle cells through MEK1/2/Erk1/2 axis activation}

Emmanouil Kyriakakis ${ }^{1}$, Agne Frismantiene ${ }^{1}$, Boris Dasen ${ }^{1}$, Dennis Pfaff ${ }^{1}$, Olga Rivero², Klaus-Peter Lesch" ${ }^{2,3,4}$, Paul Erne ${ }^{1}$, Therese J. Resink ${ }^{1}$, Maria Philippova ${ }^{1}$.

${ }^{1}$ Department of Biomedicine, Laboratory for Signal Transduction, Basel University Hospital and University of Basel, Basel, Switzerland; ${ }^{2}$ Division of Molecular Psychiatry, Center of Mental Health, University of Würzburg, Würzburg, Germany; ${ }^{3}$ Laboratory of Psychiatric Neurobiology, Institute of Molecular Medicine, I.M. Sechenov First Moscow State Medical University, Moscow, Russia; ${ }^{4}$ Department of Translational Neuroscience, School of Mental Health and Neuroscience, Maastricht University, Maastricht, The Netherlands.

Short title: T-cadherin upregulation promotes pro-survival autophagic flux

\section{Address for correspondence}

Prof. Therese Resink

Basel University Hospital, Department of Biomedicine

Laboratory for Signal Transduction

Hebelstrasse 20,

CH-4031 Basel, Switzerland.

Tel: $+41-612652422$, Fax: $+41-612652350$

Email: Therese-J.Resink@unibas.ch 


\section{ABSTRACT}

Autophagy is an evolutionary conserved intracellular catabolic process of vital importance to cell and tissue homeostasis. Autophagy is implicated in the pathogenesis of atherosclerosis but participating cells, molecular mechanisms and functional outcomes have not been fully elucidated. T-cadherin, an atypical glycosylphosphatidylinositol-anchored member of the cadherin superfamily of adhesion molecules, is upregulated on smooth muscle cells (SMCs) ${ }^{1}$ in atherosclerotic lesions. Here, using rat and murine aortic SMCs as experimental models, we surveyed the ability of T-cadherin to regulate autophagy in SMCs during serum-starvation stress. Ectopic upregulation of T-cadherin in SMCs resulted in augmented autophagy characterized by increased autophagic flux, LC3-II abundance and autophagosome formation. Analysis of signal transduction pathway effectors and use of specific pharmacological inhibitors demonstrated that T-cadherin-associated enhancement of the autophagic response to serum-deprivation was dependent on MEK1/2/Erk1/2 activation and independent of PI3K/Akt/mTORC1, reactive oxygen species or endoplasmic reticulum stress. T-cadherin upregulation on SMCs conferred a survival advantage during prolonged serumstarvation which was sensitive to inhibition of MEK1/2/Erk1/2 by PD98059 or UO126 and to blockade of autophagy by chloroquine. Loss of T-cadherin expression in SMCs diminished autophagy responsiveness and compromised survival under conditions of serum-starvation. Overall our findings have identified T-cadherin as a novel positive regulator of autophagy and survival in SMCs.

Keywords: T-cadherin, vascular smooth muscle cells, autophagy, signal transduction, unfolded protein response. 


\section{INTRODUCTION}

Autophagy is a fundamental intracellular catabolic process used by eukaryotic cells to degrade long-lived proteins and excessive or damaged organelles. Macroautophagy (hereafter referred to as autophagy) is characterized by formation of autophagosomes, double-membrane vesicles that envelop diverse cytosolic constituents and fuse with lysosomes or the vacuole to degrade their cargo. Basal autophagy represents a homeostatic mechanism for constitutive turnover of damaged organelles or cytosolic components, while its induction by stress-related signals enables cells to adapt to changing nutritional and energy demands by repurposing existing cellular components to sustain cellular metabolism, homeostasis and survival [1, 2]. Apart from supporting cellular homeostasis and adaptation to stress, autophagy is implicated in several other cellular functions including migration, proliferation, development, differentiation and tissue remodeling [1, 2]. Autophagy dysfunction is associated with diverse human pathologies such as cancer, neurodegeneration, infection, metabolic and cardiovascular diseases.

Atherosclerosis, which underlies most cardiovascular diseases, is driven by diverse extracellular and intracellular stressors, many of which (e.g. inflammatory mediators, reactive oxygen species, oxidized lipoproteins, endoplasmic reticulum stress, hypoxia, nutrient and growth factor deprivation) can trigger autophagy [3-6]. Studies based on transmission electron microscopy as well as immunoblot and immunofluorescence microscopic analysis of autophagy marker proteins (e.g. LC3-II, SQSTM1/p62 and Beclin1) have provided evidence for the occurrence of autophagy in endothelial cells, macrophages and smooth muscle cells $(\mathrm{SMCs})^{1}$ of atherosclerotic lesional tissues [3-6]. SMCs play a pivotal role throughout atherogenesis due to their ability to reversibly transition from a quiescent/contractile

\footnotetext{
${ }^{1}$ Abbreviations: AMPK, 5' adenosine monophosphate-activated protein kinase; BSA, bovine serum albumin; $\mathrm{CQ}$, chloroquine; $\mathrm{EC}$, endothelial cell; elF2 $\alpha$, eukaryotic translation initiation factor $2 \alpha$; $E R$, Endoplasmic reticulum; Erk1/2, extracellular signal-regulated kinase; E-SMC, empty vector transduced SMC; FCS, fetal calf serum; GPI, glycosylphosphatidylinositol; Grp78, 78 kDa glucose-regulated protein; Jnk, C-Jun N-terminal kinase; LC3, microtubule-associated protein 1A/1B-light chain 3; LIR, linear motif named LC3-interacting region; LY, LY294002; MAPK, mitogen activated protein kinase; MEK1/2, mitogen activated protein kinase/Erk kinase1/2; M-SMC, MOVAS murine aortic smooth muscle cell line; mTOR, mammalian target of rapamycin; NAC, N-acetyl-L-cysteine; p38 MAPK, p38 mitogen activated protein kinase; PBD, phosphate buffered saline; PD, PD98059; PI3K, phosphatidylinositol-3 kinase; rapa, rapamycin; ROS, reactive oxygen species; R-SMC, rat aortic smooth muscle cell; SMC, smooth muscle cell; Tcad+-SMC, T-cadherin-transduced SMC; TUN, tunicamycin; UPR, unfolded protein response.
} 
1 phenotype to a proliferative, migratory and synthetic phenotype [7], and autophagy has also been implicated in this behavioral flexibility [3-5] .

Cellular adhesive interactions participate in control of proliferation, migration, survival and phenotype of diverse cell types, including SMCs [8], and adhesion-dependent transduction pathways overlap considerably with those affecting autophagy [9, 10].However, in contrast to the enormous body of literature on autophagy regulation by growth factors, molecular stressors, the hormonal and nutritional environment and pharmacological agents, few studies to date have focused on relationships between the autophagic response and cell adhesion molecules. In epithelial cells there is evidence for autophagy-regulated expression of integrins [11, 12], and for both integrin-dependent suppression [13, 14] or induction of autophagy $[15,16]$. In SMCs the integrin ligand osteopontin, recognized as a marker and mediator in atherosclerotic vascular disease [17], stimulated autophagy directly through integrin/CD44 and p38 MAPK-mediated pathways [18]. Current support for relationships between autophagy and the cadherin family of adhesion molecules derives almost exclusively from studies describing effects of autophagy on cadherin expression repertoires. For instance, in squamous cell carcinoma autophagy deficiency was associated with decreased E-cadherin expression [19], in mouse embryonic fibroblasts a reduced E-cadherin expression was associated with autophagy inhibition through knockout of essential autophagy genes Atg3, Atg5, Atg9, Atg7, or Atg12 and through ectopic expression of autophagy substrate SQSTM1/p62 [19], whereas in the acute myeloid leukemia cell line THP-1 E-cadherin-mediated stabilization of $\beta$-catenin reduced autophagy [20]. In vascular endothelial cells autophagy-deficiency through Atg7 ablation resulted in loss of VE-cadherin and gain of $\mathrm{N}$-cadherin [21], while contrastingly, autophagy-induction by oxidized low density lipoprotein was associated with loss of VE-cadherin [22]. Autophagy-associated alterations in cadherin expression in SMCs have not yet been reported.

While investigations on cadherin-dependent modulation of autophagy are lacking, it is likely that cadherins might per se contribute to modification of autophagic responses. Besides mediating homophilic intercellular adhesion, cadherins are capable of heterophilic interactions with a variety of extracellular and intracellular proteins and are involved in regulating a variety of cellular processes (e.g. survival, proliferation, differentiation) analogous to those regulated by autophagy. Cadherins also affect diverse signaling pathways and effectors (e.g. PI3K/Akt/mTOR, GSK3ß, Rho GTPases, Wnt) that converge on those regulating autophagy $[9,10]$. Furthermore, a direct function for cadherins in autophagy regulation was recently identified: Gugnoni et al., demonstrated that cadherin 6 can directly bind LC3-related protein GABARAP and other autophagic proteins via LC3-interacting region (LIR) motifs within its cytoplasmic domain [23]. 
1 T-cadherin (cadherin 13, H-cadherin) is an atypical glycosylphosphatidylinositol (GPI)-

2 anchored member of the cadherin superfamily and a major cadherin expressed on SMCs. Tcadherin undergoes upregulation in vivo in vasoproliferative diseases such as atherosclerosis and restenosis [24, 25] and also in vitro under conditions/"risk factors" (e.g. hyperglycemia, oxidative stress, growth factor deprivation) encountered by SMCs in vivo [26, 27]. Functional consequences of T-cadherin upregulation in SMCs include increased proliferation and the acquisition of a dedifferentiated phenotype [28]. Signal transduction axes utilized by T-cadherin include Akt/mTORC1 and Akt/GSK3 $\beta[26,28]$ Based on several parallels between T-cadherin upregulation and autophagy in SMCs with respect to stimuli (e.g. hyperglycemia), signal transduction pathway utilization (e.g. Akt/mTORC1) and functional outcomes (e.g. phenotype switching) [3-5, 26, 28] we hypothesized that T-cadherin might regulate autophagy. Using rat and murine aortic SMCs as experimental models this study demonstrates that upregulation of T-cadherin leads to increased autophagic flux and improved survival during serum-starvation stress conditions and that these effects of $\mathrm{T}$ cadherin are mediated through MEK1/2/Erk1/2 activation.

\section{MATERIALS and METHODS}

\subsection{Cell culture, lentiviral transduction and drug treatment}

Isolation of SMCs from the descending thoracic aortae of Wistar-Kyoto rats (R-SMC) by enzymatic disaggregation and their characterization has been detailed before [28, 29]. Murine aortic SMC cell line MOVAS (M-SMC) was purchased from ATCC (CRL-2797). Stable transductants of T-cadherin-overexpressing R-SMCs and M-SMCs (Tcad+) were generated using pLVX-puro vector carrying full length human T-cadherin cDNA (empty pLVX-puro vector served as control $(E)$ ) [26, 28]. Stable transductants of T-cadherin-deficient R-SMCs (shTcad) were generated using pLKO.1-puro vector carrying rat T-cadherin shRNA (non-target shRNA served as control (shC)). R-SMCs were transduced at passages 3-4, and for both R-SMCs and M-SMCs the transduction protocols were performed on at least four independent occasions. Transductants were used at passages 6-12 following puromycin selection, and within any given experimental series Tcad+- and E-SMC or shTcad and shC transductant sets at the same passage were compared.

SMCs from the descending thoracic aortae of the previously described constitutive Cdh13 knockout mouse line (C57BI/6N) [30] and wild type littermates (respectively, Cdh13 ${ }^{-/}$-SMCs and $\mathrm{Cdh} 13^{+/+}$-SMCs) were isolated by explant culture after removal of connective tissue and adventitia and the endothelial layer under a dissecting microscope [31]. At confluence, the isolates assumed a hill-and-valley appearance typical for SMCs (Supplemental Fig. S1A). Identity as SMCs was verified by immunofluoresence staining for smooth muscle $\alpha$-actin [28] 
1 (Supplemental Fig. S1B) and by qPCR analysis of smooth muscle specific markers including 2 smooth muscle myosin heavy chain (Myh11), Transgelin (Tagln or sm22a), smooth muscle a-actin (Acta2) and caldesmon1 (Cald1) (isolates of prostate epithelial cells served as the negative control; data not shown). Cdh $13^{-/}$-SMCs and Cdh $13^{+/+}$-SMCs isolates were used between passages $4-8$, and at a matched passage for any given experiment.

Normal growth medium for all SMCs was Dulbecco's modified Eagle's medium (DMEM) supplemented with $10 \%$ fetal calf serum (FCS) and penicillin/streptomycin. To study autophagy SMCs were grown to subconfluency in normal growth medium, rinsed with PBS and then further cultured for $6 \mathrm{~h}$ under conditions of serum-deprivation (DMEM supplemented with $0.1 \%$ bovine serum albumin (BSA)). When used, chloroquine, tunicamycin, LY294002, PD98059, U0126, rapamycin or $\mathrm{N}$-acetyl-L-cysteine (all from Sigma-Aldrich Chemie, Buchs, Switzerland) were included throughout the period of serumdeprivation.

\subsection{Western blotting}

At the end of the starvation protocol cells were rinsed with PBS and lysed in buffer containing $25 \mathrm{mM}$ Tris- $\mathrm{HCl}$ (pH 7.4), $150 \mathrm{mM} \mathrm{NaCl}, 1 \% \mathrm{NP}-40,1 \mathrm{mM}$ EDTA, 5\% glycerol, phosphatase inhibitor cocktail and protease inhibitor cocktail (both from Roche Diagnostics (Schweiz) AG, Risch-Rotkreuz, Switzerland) to preserve protein phosphorylation status. Protein concentrations were determined using the BCA Protein Assay Kit (ThermoScientific (Schweiz) AG, Reinach, Switzerland). Crude cell protein lysates (10-15 $\mu \mathrm{g} /$ lane) were subjected to standard SDS-polyacrylamide gel electrophoresis under reducing conditions and electro-blotted onto nitrocellulose or PVDF membranes. Membranes were variously immune-probed using primary antibodies against the following proteins/epitopes: T-cadherin (R\&D Systems Europe Ltd., Abingdon, UK), GAPDH, (Abcam, Cambridge, UK), LC3B (rabbit

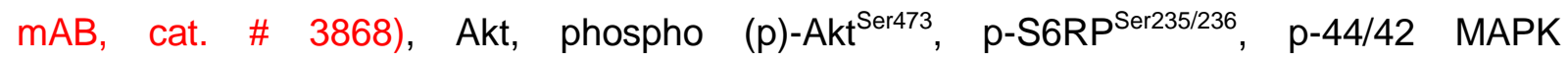

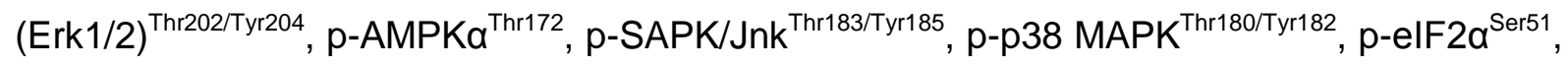
p-GSK3 $\beta^{\text {Ser9 }}$ (Cell Signalling Technologies, New England Biolabs GMBH, Frankfurt, Germany), Grp78 (Santa Cruz Biotechnology Inc., Heidelberg, Germany), tubulin (SigmaAldrich Chemie, Buchs, Switzerland). Secondary HRP-conjugated anti-species specific IgGs were from Southern Biotechnology (BioReba AG, Reinach, Switzerland). GAPDH, Akt or tubulin were variously used as loading controls. Representative blots following signal detection using the Bio-RadMolecular Imager Gel Doc XR+ system (Bio-Rad Laboratories, Hercules, CA, USA) are shown. Densitometric quantification of signals was performed using ImageJ (NIH, Bethesda, MD, USA) or Image Lab (Bio-Rad) software. 


\subsection{Detection of autophagosome formation}

Autophagosome formation in cells was detected using either a CYTO-ID Autophagy Detection Kit (CYTO-ID®: Enzo Life Sciences ENZ-51031-K200, PA, USA), which employs the use of CYTO-ID fluorescent reagent that specifically labels autophagic vacuoles and colocalizes with LC3, or by immunofluorescence staining for specific autophagosome membrane markers LC3B and/or p62/SQSTM1. SMCs in normal growth medium were seeded onto gelatin-coated coverslips into triplicate wells of 24 -well plates ( $4 \times 10^{4}$ cells/well), allowed to adhere overnight and then subjected to the serum-deprivation protocol. For CYTO-ID® detection of autophagosomes live SMCs were stained in-well with according to the manufacturer's instructions. Immunofluorescence staining for LC3B or p62/SQSTM1 was performed on methanol fixed and permeabilized (0.3\% Triton $\left.{ }^{\mathrm{TM}} \mathrm{X}-100\right)$ SMCs following standard protocols. Primary antibodies were anti-LC3B (rabbit mAB, Cell Signalling Technologies cat.\# 3868) and anti-p62/SQSTM1 (murine mAB, Abcam cat. \# ab56416). Antispecies specific secondary Alexa Fluor 488-conjugated IgGs (Invitrogen, MA, USA) were used for detection. Nuclei were counter-stained with Hoechst 33342 dye. Images were acquired under an Olympus IX83 fluorescent microscope, equipped with a DP80 camera and CellSens software (all from Olympus) or under an LSM-710 confocal laser scanning microscope equipped with Zen software (all from Zeiss). Autophagic vesicle accumulation was quantitated using ImageJ software (Trainable Weka Segmentation plugin was used), and expressed as number of autophagic vesicles (autophagosomes) per cell.

\subsection{Cell survival assay}

SMCs in normal growth medium were seeded into quadruplicate wells of 96-well plates $\left(3 \times 10^{3}\right.$ cells/well), and allowed to adhere overnight. After rinsing with PBS (day 0$)$ cells were further cultured for up to 3 days in serum-free medium (DMEM containing $0.1 \%$ BSA). To estimate cell survival wells were rinsed gently with PBS and the remaining adherent cells quantitated by enzymatic detachment $(0.25 \%$ trypsin/1mM EDTA in PBS) and counting in a Coulter counter. Cell survival is expressed as the number of adherent cells relative to that at day 0 (arbitrarily taken as 100\%).

\subsection{Analysis of apoptosis by flow cytometry}

Following serum-starvation for 3 days without or with inclusion of MEK1/2 inhibitor, SMCs were harvested and pooled with their respective supernatant, so as to include any detached apoptotic SMCs. SMCs were pelletted by centrifugation at $1200 \mathrm{rpm}$ at $4^{\circ} \mathrm{C}$ and washed twice in chilled $\left(\approx 4^{\circ} \mathrm{C}\right)$ PBS. Cells were stained with fluorescein isothiocyanate-conjugated Annexin V protein (FITC-Annexin V) and propidium iodide (PI) using FITC-Annexin V 
1 Apoptosis Detection Kit (BD Biosciences-Europe, Allschwil, Switzerland) according to the

2 manufacturer's protocol. Acquisition and analysis were performed using BD ACCURI ${ }^{\mathrm{TM}}$ C6

3 flow cytometer (BD Biosciences).

\subsection{Statistical analysis}

Every experimental series was performed on at least 3 separate occasions and unless otherwise stated results are given as mean \pm S.D. GraphPad Prism 5.0 (GraphPad Software, San Diego, CA, USA) was used for statistical analysis. $P$-values were calculated by two tailed Student's t-test for single comparisons and by one-way analysis of variance (ANOVA) followed by Tukey post-hoc testing for multiple comparisons. A 2-tailed probability value $<0.05$ was considered as threshold of significance. Survival curves were analysed by nonlinear regression with least squares second order polynomial curve fitting (quadratic) with extra sum-of-squares $\mathrm{F}$ test as comparison method. $P<0.05$ was considered as threshold of significance.

\section{RESULTS}

3.1. T-cadherin upregulation on SMCs increases autophagic flux during growth factor deprivation

The chief experimental models used in this study are SMCs isolated from rat aortae (R-SMC) and the murine aortic SMC cell line MOVAS (M-SMC), both stably transduced with vector carrying human T-cadherin (Tcad+-SMC) or control empty vector (E-SMC). Representative immunoblots of T-cadherin protein (Mr of $105 \mathrm{kDa}$ and $130 \mathrm{kDa}$ for mature and prepro proteins, respectively) in whole cell lysates are shown in Figure $1(A, B)$.

Unlike unicellular organisms, metazoans depend on extracellular growth factors to regulate uptake and digestion of environmental nutrients, and the withdrawal of growth factors or culture of cells in serum-free medium initiates autophagy [32]. Initial experiments comparing autophagy in Tcad+ and control E-SMCs under normal growth (serum-containing) conditions found that Tcad+-SMCs have higher levels of constitutive autophagy, based on elevated levels of autophagy marker LC3-II (Supplemental Fig. S2). Subsequent experiments focussed on examining the impact of T-cadherin on autophagy during serum-starvation: culture under conditions of serum-deprivation is commonly used to render a state of quiescence and/or synchrony in SMCs, but effects of serum-deprivation per se on autophagy in this cell type have never been investigated.

Serum-starvation induced autophagy in SMCs, and LC3-II increased to higher levels in Tcad+-SMCs than in control E-SMCs (Supplemental Fig. S2 and Fig. 1A, B), suggesting that 
T-cadherin upregulation in SMCs enhances their autophagic response to serum-deprivation. Since formation of LC3-II is transient during stimulus-induced autophagy and the protein can be rapidly degraded in the lysosome an increase in LC3-II might also be indicative of decreased autophagy [33]. Therefore, to more accurately assess autophagic flux, SMCs were subjected to serum-deprivation in the presence or absence of chloroquine (CQ), a lysosomotropic agent which inhibits lysosomal activity and lysosomal degradation of LC3-II [34]. CQ induced a further increase in abundance of LC3-II above that of serum-deprivation alone in both E- and Tcad+-SMC, with levels of LC3-II remaining elevated in Tcad+-SMCs (Fig. 1A, B), supporting higher autophagic flux in Tcad+-SMCs.

To obtain further evidence for stimulatory effects of T-cadherin upregulation on autophagy during serum-starvation we investigated the accumulation of autophagosomes. Use of the CYTO-ID ${ }^{\circledR}$ Autophagy Detection Kit to monitor autophagy in live cells after the $6 \mathrm{~h}$ period of serum-deprivation demonstrated an increased number of autophagic vacuoles in Tcad+SMCs (Fig. 1C, D). CQ caused a significant increase in autophagosome accumulation in Eand Tcad+-SMCs and autophagosome numbers/cell remained elevated in Tcad+ (Fig. 1C, D). To confirm these findings, immunofluorescence staining two specific autophagosomal membrane markers p62/SQSTM1 or LC3B in rat SMC transductants fixed after serumstarvation was performed. Compared with E-SMCs the Tcad+-SMCs exhibited a greater number of autophagic puncta, and this difference remained upon inclusion of $C Q$ (Fig. 2). Together these data support that T-cadherin upregulation promotes autophagosome formation rather than disruption of its maturation into the autophagolysosome.

As a positive control for our evaluation of serum-starvation-induced autophagy SMCs were incubated in amino acid/serum free (SF+aaf) starvation buffer (Earle's Balanced Salt Solution (EBSS)), which is known to induce robust autophagy. Interestingly, Tcad+-SMCs exhibited heightened autophagic flux also under this condition (Supplemental Fig. S3). Inclusion of CQ with EBSS increased both LC3-II protein abundance Supplemental Fig. S3A) and autophagosome accumulation (Supplemental Fig. S3B). In the absence of CQ, levels of LC3II in EBSS were lower than in serum-free medium, which probably reflects a more rapid induction of autophagic flux by EBSS.

\subsection{T-cadherin-enhanced autophagy is independent of PI3K/Akt/mTOR pathway signaling}

A key regulator of autophagy is mammalian target of rapamycin (mTOR): activation of mTORC1 inhibits autophagy whereas the inhibition of mTORC1 leads to autophagy activation [35, 36]. However, Tcad+-SMCs exhibit Akt/mTORC1 pathway hyperactivity under the experimental conditions of serum-deprivation as indicated by their elevated (vs. E-SMCs) phosphorylation status of mTORC1/p70S6K-target protein S6RP (Fig. 3A, B E, F and 
1 Supplementary Fig. S4A, and [26]). Furthermore, neither mTOR inhibition by rapamycin 2 (blots in Fig. 3A, E) nor PI3K inhibition by LY294002 (blots in Fig. 3B, F) resulted in a further accumulation of LC3-II protein in E- and Tcad+-SMCs or a normalization of their autophagy responses (quantification in Fig. 3D, H)

AMPK, which directly inhibits TORC1 activity by phosphorylating RAPTOR [35, 36], can also be excluded as a triggering mediator for T-cadherin-associated enhancement of the autophagic response since levels of p-AMPK were not different between E- and Tcad+SMCs (Supplementary Fig. S4B).

Akt target GSK3 $\beta$ plays a positive role in autophagy under conditions of serum-starvation [32]. Pharmacological inhibition or genetic silencing of GSK3 $\beta$ has been demonstrated to block autophagy, whereas activation of GSK3 $\beta$ promoted autophagy [37]. However, as shown in Figure 3B, the increased autophagic response of Tcad+-SMCs under conditions of serum-deprivation was concurrent with an increased state of GSK3 $\beta$ inactivation (i.e. levels of $p$-GSK3 $\beta^{\text {ser9 }}$ higher in Tcad+-SMCs vs. E-SMCs). Moreover, indirect activation of GSK3 $\beta$ (i.e. reduction in p-GSK3 $\beta^{\text {ser9 }}$ levels) through LY294002-mediated inhibition of PI3K/Akt (Fig. 3B) was also not associated with alterations of autophagy in either E- or Tcad+ SMCs.

Taken together, these data support that T-cadherin-enhanced autophagy in SMCs during serum-deprivation is not dependent on the PI3K/Akt/mTORC1 pathway of autophagy regulation.

\subsection{T-cadherin-enhanced autophagy is independent of reactive oxygen species}

Induction of autophagy may be directly or indirectly related to oxidative stress and the production of reactive oxygen species (ROS) under conditions of serum-starvation [38]. Inclusion of ROS scavenger $\mathrm{N}$-acetylcysteine throughout the period of serum-starvation diminished the autophagy response in both E- and Tcad+-SMCs, but failed to normalize the higher response of Tcad+-SMCs (Fig. 3C, D, G, H). Thus, although ROS participate in the induction of autophagy in SMCs under conditions of serum-deprivation, the T-cadherinassociated enhancement of the autophagic response is independent of ROS.

\subsection{T-cadherin-enhanced autophagy is independent of endoplasmic reticulum stress} protein response (UPR,) the major endoplasmic reticulum (ER) stress pathway [4, 39]. Our 
1 Grp78 and p-elF2 $\alpha$ (downstream of the IRE1 $\alpha$ and PERK branch of the UPR) in Tcad+2 SMCs (Supplementary Fig. S4D). Activities of the pro-apoptosis stress kinases Jnk and p38

3 MAPK (downstream of the IRE1a branch of the UPR) were also reduced in Tcad+-SMCs 4 (Supplementary Fig. S4C). These data imply that the increased autophagy response of 5 Tcad+-SMCs is not driven by UPR. To examine this further, we included the prototypical ER stress-inducing agent tunicamycin during the period of serum-deprivation. Tunicamycin increased levels of Grp78 and p-elF2 $\alpha$, but to a lower magnitude in Tcad+-SMCs ( 1.4-fold) than in the E-SMCs ( 1.9-fold) (Fig. 4A, B). Further, induction of UPR by tunicamycin in SMCs was accompanied by a reduction in LC3-II abundance in both E- and Tcad+-SMCs

(Fig. 4A, B). Together, these data provide evidence that neither the induction of autophagy in SMCs during serum-starvation nor the T-cadherin-associated enhancement of the autophagy response are driven by activation of UPR.

3.5. Erk1/2 activation is important for induction of autophagy in SMCs under conditions of serum-deprivation

Mitogen activated protein kinases (MAPKs), namely p38 MAPK, c-Jun N-terminal kinase (Jnk) and extracellular signal-regulated kinase (Erk1/2), play a variety of roles in controlling the balance of autophagy and apoptosis [36]. Initial screening for the activation (phosphorylation) status of these MAPKs in cultures of rat aortic E- and Tcad+-SMCs under conditions of serum-deprivation showed reduced activities of p38 MAPK and Jnk concomitant with increased Erk1/2 activation (Supplementary Fig. S4C) in Tcad+-SMCs. Since much evidence supports a positive role for Raf/MEK/Erk pathway activation in autophagy [36, 40] we assessed its involvement in our experimental setting by inclusion of the Erk1/2 upstream kinase (MEK1/2) inhibitor PD98059 during the period of serumdeprivation. PD98059 repressed autophagic flux in E- and Tcad+-SMCs as evidenced by the decrease in both LC3-II levels (Fig. 5A, B) and autophagosome numbers (Fig. 5C, D). Inhibitory effects of PD98059 were more prominent in T-cad+-SMCs, resulting in near normalization of the level of autophagic flux between E- and Tcad+-SMCs in the rat SMC model (R-SMC) and complete normalization in the murine SMC model (M-SMC). UO126, another highly selective MEK1/2 inhibitor, was tested in the R-SMC transductants: inhibitory effects of U0126 on serum-deprivation induced elevation of LC3-II levels and autophagosome numbers were similar to those of PD98059 (Supplemental Fig. S5). Together, these data imply that MEK1/2/Erk1/2 pathway activation is necessary for autophagic flux in SMCs under conditions of serum-deprivation and that enhanced MEK1/2/Erk1/2 pathway activation underlies T-cadherin-upregulation associated enhancement of autophagy. 


\subsection{T-cadherin-enhanced autophagy is associated with improved cell survival}

Functional autophagy represents an important survival strategy during nutrient or growth factor deprivation [32]. Culture of SMCs transductants for 3 days under conditions of serumdeprivation demonstrated that both rat and murine aortic Tcad+-SMCs were more robust than their control E-SMC counterparts (Fig. 6A, B). Support for the different survival capacities of $\mathrm{E}$ and Tcad+ transductants during serum-deprivation was obtained by FITCAnnexin V/propidium iodide (PI) dual color flow cytometric analysis of cell apoptosis in RSMCs. The percentages of early and late apoptotic cells in Tcad+-SMCs (16.9 \pm 2.8 and 2.6 \pm 0.4 , respectively) were significantly lower $(P<0.01)$ than in the E-SMCs $(25.1 \pm 1.9$ and $7.2 \pm 0.5$, respectively) (Supplemental Fig. S6).

Survival of SMCs was identically compromised in the E- and Tcad+-SMCs transductants by inclusion of autophagy inhibitor CQ (Fig. 6A, B). The dose of $30 \mu \mathrm{M}$ was selected because it achieved effective inhibition of LC3-II degradation within $6 \mathrm{~h}$ of serum-deprivation; lower doses $(10$ and $5 \mu \mathrm{M})$ required a prolonged $(24 \mathrm{~h}$ ) incubation period (Supplemental Fig. S7A). To control for CQ cytotoxicity SMCs were cultured for 3 days in the presence of CQ under normal serum-containing growth conditions: a reduction/plateau in cell numbers first became evident on day 3 and only for $30 \mu \mathrm{M} \mathrm{CQ}$ (Supplemental Fig. S7B). SMC survival under serum-free conditions was comparably reduced at all tested CQ doses (Supplemental Fig. S7C). These data support that reductive effects of autophagy inhibitor CQ on SMC survival during serum-deprivation are not due to cytotoxicity per se. Inclusion of MEK1/2 inhibitor PD98059 during the 3 day culture period reduced SMC survival to comparably low levels in E- and Tcad+-SMCs (Fig. 6A, B). The dose of PD98059 used (10 $\mu \mathrm{M})$ was selected because it effectively inhibited Erk1/2 activation and was not cytotoxic (Supplemental Fig. S8A, B). At a lower dose $(2 \mu \mathrm{M})$ PD98059 failed to block Erk1/2 activation and did not reduce SMC survival (Supplemental Fig. S8C). MEK1/2 inhibitor UO126 (5 $\mu \mathrm{M}$; an effective, non-cytotoxic dose) also reduced SMC survival to comparably low levels in E- and Tcad+-SMCs (Fig. 6C).

Taken together, these data support that autophagy in SMCs during serum-deprivation promotes cell survival, MEK1/2 activation is required for this protection, and upregulation of T-cadherin endows SMCs with an improved survival capacity.

\subsection{T-cadherin depletion blunts the autophagy response of SMCs to serum-starvation}

To confirm and consolidate the findings that T-cadherin enhances autophagy and survival in SMCs during serum-deprivation we examined the consequence of T-cadherin loss-offunction. Two experimental models were used, namely aortic SMC isolates from a constitutive Cdh13 knockout mouse line (Cdh13/-SMC) and the wild type control $\left(\mathrm{Cdh} 13^{+/+}\right.$ 
SMC), and rat aortic SMCs stably transduced with lentivector carrying rat T-cadherin shRNA (shTcad-SMC) or non-target shRNA as control (shC-SMC). Representative immunoblots for T-cadherin are shown in Figure 7. In both knockout (Fig. 7A, C) and shRNA-mediated silencing (Fig. 7B, D) models, the loss of T-cadherin attenuated autophagic flux as evidenced by a reduction in accumulation of LC3-II protein and autophagosomes. Attenuation was more prominent in Cdh13 ${ }^{-/}$-SMCs (by $\approx 80 \%$ vs. Cdh $13^{+/+}-$SMCs) than in the shTcad-SMCs (by $\approx$ $40 \%$ vs. shC-SMCs), likely reflecting incomplete shRNA-mediated silencing of T-cadherin in the latter (Fig.7B). Loss of T-cadherin function also negatively impacted Erk1/2 activity, and as for autophagic flux the inhibitory effect was much more marked in Cdh1 $13^{-/}$-SMCs than shTcad-SMCs (Fig. 7A, B). The consequence of T-cadherin loss-of-function for survival under conditions of serum-deprivation was tested in the knockout model. Assays for cell enumeration (Fig. 8A) and apoptosis (Fig. 8B) demonstrated that Cdh13 ${ }^{-/}$-SMCs exhibited a

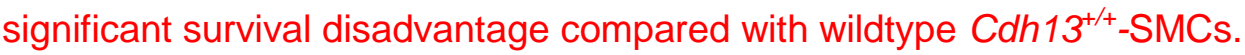

DISCUSSION

The present study demonstrates a novel role for T-cadherin in regulating autophagy in SMCs. Ectopic upregulation of T-cadherin augmented autophagy under conditions of serumwithdrawal as characterized by increased LC3-II abundance, autophagic flux and autophagosome formation. In contrast, shRNA-mediated depletion of T-cadherin or Cdh13 gene ablation attenuated the autophagy response of SMCs to serum-starvation. Activation of MEK1/2/Erk1/2 is a key event in the elevated autophagic response of $T$-cadherin overexpressing SMCs to serum-deprivation. Further, T-cadherin upregulation confers a survival advantage in the face of prolonged serum-withdrawal, and this was sensitive to inhibition of MEK1/2/Erk1/2 by PD98059 or U0126 as well as blockade of autophagy by CQ. T-cadherin-deficient SMCs exhibit a survival disadvantage under conditions of serumstarvation.These findings, summarized in Figure 9, identify T-cadherin as a novel positive regulator of autophagy and survival in SMCs.

Although the occurrence of autophagy in SMCs in the context of atherosclerosis has been recognized for decades, many gaps still exist in our knowledge concerning the participating molecular mechanisms and impact on disease progression. Depending on exogenous factors as well as the cell's ability to handle the stress to which it is exposed autophagy can exert detrimental or protective effects. For example, SMC death occurred in association with induction of autophagy by tumor necrosis factor a [41] or angiotensin II [42] while the inhibition of autophagy in plaque-derived SMCs by insulin-like growth factor promoted survival [41]. Conversely, SMC survival accompanied autophagy induced by stress stimuli such as hypoxia [43, 44], various oxidized lipids [45-47] or free cholesterol overload [48]. 
1 Activation of autophagy by hypoxia [44], platelet-derived growth factor [49], advanced glycation end products [50] and the developmental morphogen secreted protein sonic hedgehog [51] have also been associated with SMC proliferation. Further, defective autophagy, elicited by deletion of the essential autophagy gene Atg7 in SMCs, resulted in cell cycle arrest, reduced proliferation, accelerated senescence and cellular hypertrophy [52]. Although SMCs in advanced atherosclerotic lesions suffer pro-autophagic conditions of restricted nutrient and growth factor supply [3-6], there are no investigations on autophagy in SMCs during serum-starvation per se. Our study suggests that autophagy in SMCs under conditions of serum/growth factor-deprivation may function as a protective, catabolic mechanism to safeguard against associated cellular distress.

The central finding of this study, namely that T-cadherin upregulation promotes pro-survival autophagic flux, is in accordance with our previous studies demonstrating that T-cadherin is a marker of vascular cell activation and stress. T-cadherin upregulation on SMCs and endothelial cells (ECs) is observed in vivo during atherosclerosis [24] and in vitro following exposure to stress conditions associated with development of atherosclerosis (e.g. inflammatory, oxidative, hyperglycaemic, hyperinsulinaemic stress, prolonged growth factor/serum-deprivation) [26, 53-55]. Circulating levels of T-cadherin harbored on ECderived microparticles are elevated during early atherosclerosis [23] but decreased in association with severity of coronary lesion severity and acute coronary syndrome [56]. Tcadherin upregulation on SMCs and ECs impacts their phenotype (promoting acquisition of dedifferentiated and pro-angiogenic phenotypes, respectively) with associated alterations in proliferative, adhesive and migratory capacities [28, 57]. T-cadherin upregulation improves cell survival under stress conditions such as oxidative stress [53], ER stress [58] and serumstarvation (this study and [53]). The ability of T-cadherin to facilitate pro-survival autophagy further underscores the role for this protein in preserving vascular cell function during proatherogenic stress. Interestingly, this study also noted the occurrence of elevated basal/constitutive autophagy (i.e. under normal serum-containing growth conditions) in Tcad+-SMCs, which might reflect their acquisition of a highly dedifferentiated phenotype and associated prominent decay of SMC-differentiation markers (smooth muscle alpha-actin, smooth muscle myosin heavy chain, h-caldesmon) [28]. Such an interpretation would be in line with previous findings by Salabei et al., [49] that PDGF-promoted transition from the contractile to the dedifferentiated phenotype was accompanied by increased (and mTORindependent) autophagy. These authors suggested that dedifferentiation-associated autophagy might serve as a major proteolytic mechanism to remove contractile proteins during phenotype transition. 
1 Signaling pathways implicated as mediators of autophagy in SMCs are diverse and dependent on the stimulus. For example, autophagy induction by advanced glycation end products was mediated through activation of Erk and inhibition of Akt [50]. Induction of autophagy by tumor necrosis factor a induced autophagy occurred through Jnk activation and Akt inhibition [41]. Oxidative stress-associated stimulation of autophagy by 7ketocholesterol, 4-hydroxy-trans-2-nonenal or hypoxia involved generation of hydrogen peroxide [47], activation of ER stress/Jnk [45] or activation of AMPKa1 [43], respectively. Osteopontin induced autophagy via integrin/CD44 and p38 MAPK [18]. The signaling pathways mediating autophagy induced by platelet-derived growth factor [49] or angiotensin II [59] were not identified, although involvement of mTOR and AMPK were excluded. Here, our pharmacological approach to identify signaling mediators for autophagy in SMCs during serum-deprivation identified a requirement for MEK1/2/Erk1/2 activation and excluded participation of mTORC1 and AMPK. Interestingly, MEK1/2 inhibitor PD98059 normalized pErk1/2 to equivalently low levels in the murine E- and Tcad+-SMC transductants, but not in the rat transductants; $\mathrm{p}$-Erk1/2 remained at a higher level in the Tcad+-SMCs, also at higher doses (up to $40 \mu \mathrm{M}$, data not shown). A similar observation was made in R-SMCs for the MEK1/2 inhibitor UO126. This suggests existence of alternative MEK1/2-independent pathways that maintain Erk $1 / 2$ activity at low but functional levels when T-cadherin is upregulated.

mTOR plays an important role in regulating autophagy, and mTOR inhibitor rapamycin (or analogs) are used frequently to induce autophagy [35, 36]. However, it is recognized that the effectiveness of rapamycin on inducing autophagy in mammalian cells is dependent on the cell type, despite its ubiquitous inhibition of mTOR activity [35]. For example, in a panel of glioma cell lines, rapamycin effectively induced autophagy in U87-MG and T98G cells but was not sufficient to induce autophagy in U373-MG cells [60], while amongst bladder carcinoma, osteosarcoma and neuroblastoma cell lines, rapamycin failed to induce autophagy in the neuroblastoma cell lines [55]. Although rapamycin effectively inhibited mTOR signaling and proliferation in SMCs (this study and [28]) it failed to induce autophagy, a finding that is in line with other studies. Li et al., found induction of autophagy in SMCs under serum-free conditions only at rapamycin doses $(>10 \mu \mathrm{M})$ several orders of magnitude higher than those $(<10 \mathrm{nM})$ required to inhibit mTOR signaling and proliferation [61]. Verheye et al., found that under serum-containing conditions rapamycin (10 $\mu \mathrm{M})$ induced robust autophagy in macrophages, but failed to induce an autophagis response in SMCs [62]. This macrophage-selective activation of autophagy was confirmed by ex vivo TEM analysis of cell type autophagy after 3 days exposure of explanted vascular tissue segments to rapamycin [62]. Two other studies that used SMCs loaded with 7-keto-cholesterol [47] or 
$\mu \mathrm{M})$ higher than that used (250 nM) in our current study. Collectively, such data suggest that as a cell type, SMCs are relatively resistant to induction of autophagy by rapamycin.

Quite how GPI-anchored T-cadherin on the cellular membrane regulates autophagy remains

4 entirely speculative. We may consider potential autophagy-facilitating characteristics of Tcadherin. Firstly, plasma membrane T-cadherin is located within lipid raft domains [63]: autophagosomes can originate from the plasma membrane and there is evidence for involvement of lipid rafts in autophagosome formation [64]. In this context, it is interesting that caveolin-1, a major raft domain resident protein, was found to interact with ATG5 and ATG12, and their active complex ATG12-ATG5, thereby suppressing the formation and activity of ATG12-ATG5 conjugate and subsequent autophagy in lung epithelial cells [56]. We might speculate upregulation of GPI-anchored glycoprotein such as T-cadherin leads to alterations in raft domain fluidity or protein composition and/or interactions that facilitate dissociation of caveolin-1/autophagy protein complexes and thereby trigger autophagy. Secondly, T-cadherin, like many other cadherin family members, contains a putative LIR domain [65]: LIR domains mediate selective interaction of proteins with the autophagic machinery [66] and several LIR-motif-containing proteins are involved in autophagosome formation, transport and maturation [67]. In their recent study on thyroid cancer cells Gugnoni et al., demonstrated that type 2 cadherin 6 restrained autophagy through direct LIR-mediated interaction of its cytoplasmic domain with GABARAP and BNIP3 and/or BNIP3L [23]. A direct interaction of T-cadherin with autophagy proteins through its putative LIR domain would seem rather unlikely because it possesses neither transmembrane nor cytoplasmic domains. Importantly, Gugnoni et al., also showed that promotion of autophagy following silencing of cadherin 6 in thyroid tumour cells was accompanied by upregulation of epithelial marker cadherin 16 , which has a truncated cytoplasmic domain, and a partial reversion of epithelialto-mesenchymal phenotype transition. Although modulation of T-cadherin gene and protein expression in SMCs also leads to alterations in phenotype transition [28,68], a concomitant occurrence of cadherin switching has not been investigated. However, and in order to reconcile the opposing effects of cadherin 6 and T-cadherin on autophagy, and by analogy with cadherin 6/cadherin 16 switching in thyroid tumour cells [23], we might speculate that Tcadherin upregulation in SMCs and its ability to promote autophagy is associated with concomitant downregulation of some transmembrane cadherin normally competent to bind/sequester autophagy machinery components, thereby allowing for activation of autophagy. Thirdly, T-cadherin may sense bioenergetic perturbations through its ability to interact with receptors involved in nutrient-sensing and autophagy regulation: investigations in EC and tumor cells have demonstrated direct and/or indirect crosstalk between T-cadherin and growth factor receptors such as fibroblast growth factor receptor, epidermal growth factor receptor and insulin receptor [54, 69-71]. 


\section{CONCLUSIONS}

2 In conclusion, our study has identified the existence of cadherin-based autophagy regulation

3 in SMCs. The data supports that in SMCs T-cadherin has protective functions under 4 conditions of bioenergetic distress which prevail in atherosclerotic lesions, and that 5 upregulation of T-cadherin serves to harness the support of the autophagy machinery in 6 order to improve cell survival.

\section{$9 \quad$ Acknowledgements}

10 This work was supported by the Swiss National Science Foundation (grant no. 11 310030_159589 to TJR), Stiftung für Herz- und Kreislaufkrankheiten (to TJR and MP) and 12 Swiss Heart Foundation (to MP). The generation and characterization of the $\mathrm{Cdh}^{13^{-}}$mouse 13 line was supported by the the European Community (EC: AGGRESSOTYPE FP7/No. 14602805 to KPL and OR), Fritz Thyssen Foundation (Az. 10.13.1185 to KPL) and 5-100 15 Russian Academic Excellence Project (to KPL).

\section{Author contributions}

17 EK, TJR, and PE designed the study and experiments. EK, AF, BD, DP, OR conducted 18 experiments. EK, TJR and MP analysed data; BD generated lentiviral vectors and viral 19 particles; K-PL, OR generated the Cdh $13^{-/}$mouse line; EK and TJR drafted the manuscript. 20 All authors critically read, edited draft versions and approved the final manuscript. 


\section{REFERENCES}

[1] Z. Yang, D.J. Klionsky, Eaten alive: a history of macroautophagy, Nature cell biology 12(9) (2010) 814-22.

[2] N. Mizushima, M. Komatsu, Autophagy: renovation of cells and tissues, Cell 147(4) (2011) 728-41.

[3] G.R. De Meyer, M.O. Grootaert, C.F. Michiels, A. Kurdi, D.M. Schrijvers, W. Martinet, Autophagy in vascular disease, Circulation research 116(3) (2015) 468-79.

[4] J.K. Salabei, B.G. Hill, Implications of autophagy for vascular smooth muscle cell function and plasticity, Free radical biology \& medicine 65 (2013) 693-703.

[5] S. Tai, X.Q. Hu, D.Q. Peng, S.H. Zhou, X.L. Zheng, The roles of autophagy in vascular smooth muscle cells, International journal of cardiology 211 (2016) 1-6.

[6] S. Lavandero, R. Troncoso, B.A. Rothermel, W. Martinet, J. Sadoshima, J.A. Hill, Cardiovascular autophagy: concepts, controversies, and perspectives, Autophagy 9(10) (2013) 1455-66.

[7] M.R. Bennett, S. Sinha, G.K. Owens, Vascular Smooth Muscle Cells in Atherosclerosis, Circulation research 118(4) (2016) 692-702.

[8] E.P. Moiseeva, Adhesion receptors of vascular smooth muscle cells and their functions, Cardiovasc Res 52(3) (2001) 372-86.

[9] E.A. Nollet, C.K. Miranti, Integrin and adhesion: regulation of autophagy and mitophagy, in: Y. Bailley (Ed.), Autophagy - A Double-Edged Sword - Cell Survival or Death?, InTech, 2013, pp. pp. 465-485.

[10] R. Lock, J. Debnath, Extracellular matrix regulation of autophagy, Current opinion in cell biology 20(5) (2008) 583-8.

[11] C.M. Kenific, S.J. Stehbens, J. Goldsmith, A.M. Leidal, N. Faure, J. Ye, T. Wittmann, J. Debnath, NBR1 enables autophagy-dependent focal adhesion turnover, The Journal of cell biology 212(5) (2016) 577-590.

[12] V. Tuloup-Minguez, A. Hamai, A. Greffard, V. Nicolas, P. Codogno, J. Botti, Autophagy modulates cell migration and beta1 integrin membrane recycling, Cell cycle 12(20) (2013) 3317-28.

[13] Q. He, B. Huang, J. Zhao, Y. Zhang, S. Zhang, J. Miao, Knockdown of integrin beta4induced autophagic cell death associated with P53 in A549 lung adenocarcinoma cells, The FEBS journal 275(22) (2008) 5725-32.

[14] S.L. Lomonaco, S. Finniss, C. Xiang, H.K. Lee, W. Jiang, N. Lemke, S.A. Rempel, T. Mikkelsen, C. Brodie, Cilengitide induces autophagy-mediated cell death in glioma cells, Neuro-oncology 13(8) (2011) 857-65.

[15] M.J. Edick, L. Tesfay, L.E. Lamb, B.S. Knudsen, C.K. Miranti, Inhibition of integrinmediated crosstalk with epidermal growth factor receptor/Erk or Src signaling pathways in 
autophagic prostate epithelial cells induces caspase-independent death, Molecular biology of the cell 18(7) (2007) 2481-90.

[16] V. Tuloup-Minguez, A. Greffard, P. Codogno, J. Botti, Regulation of autophagy by extracellular matrix glycoproteins in HeLa cells, Autophagy 7(1) (2011) 27-39.

[17] T. Wolak, Osteopontin - a multi-modal marker and mediator in atherosclerotic vascular disease, Atherosclerosis 236(2) (2014) 327-37.

[18] Y.H. Zheng, C. Tian, Y. Meng, Y.W. Qin, Y.H. Du, J. Du, H.H. Li, Osteopontin stimulates autophagy via integrin/CD44 and p38 MAPK signaling pathways in vascular smooth muscle cells, Journal of cellular physiology 227(1) (2012) 127-35.

[19] L. Qiang, B. Zhao, M. Ming, N. Wang, T.C. He, S. Hwang, A. Thorburn, Y.Y. He, Regulation of cell proliferation and migration by $\mathrm{p} 62$ through stabilization of Twist1, Proceedings of the National Academy of Sciences of the United States of America 111(25) (2014) 9241-6.

[20] K. Kuhn, W. Romer, Considering autophagy, beta-Catenin and E-Cadherin as innovative therapy aspects in AML, Cell death \& disease 6 (2015) e1950.

[21] K.K. Singh, F. Lovren, Y. Pan, A. Quan, A. Ramadan, P.N. Matkar, M. Ehsan, P. Sandhu, L.E. Mantella, N. Gupta, H. Teoh, M. Parotto, A. Tabuchi, W.M. Kuebler, M. AlOmran, T. Finkel, S. Verma, The essential autophagy gene ATG7 modulates organ fibrosis via regulation of endothelial-to-mesenchymal transition, The Journal of biological chemistry 290(5) (2015) 2547-59.

[22] D.H. Wei, X.Y. Jia, Y.H. Liu, F.X. Guo, Z.H. Tang, X.H. Li, Z. Wang, L.S. Liu, G.X. Wang, Z.S. Jian, C.G. Ruan, Cathepsin L stimulates autophagy and inhibits apoptosis of ox-LDLinduced endothelial cells: potential role in atherosclerosis, International journal of molecular medicine 31(2) (2013) 400-6.

[23] M. Gugnoni, V. Sancisi, G. Gandolfi, G. Manzotti, M. Ragazzi, D. Giordano, I. Tamagnini, M. Tigano, A. Frasoldati, S. Piana, A. Ciarrocchi, Cadherin-6 promotes EMT and cancer metastasis by restraining autophagy, Oncogene (2016).

[24] D. Ivanov, M. Philippova, J. Antropova, F. Gubaeva, O. Iljinskaya, E. Tararak, V. Bochkov, P. Erne, T. Resink, V. Tkachuk, Expression of cell adhesion molecule T-cadherin in the human vasculature, Histochemistry and cell biology 115(3) (2001) 231-42.

[25] E. Kudrjashova, P. Bashtrikov, V. Bochkov, Y. Parfyonova, V. Tkachuk, J. Antropova, O. Iljinskaya, E. Tararak, P. Erne, D. Ivanov, M. Philippova, T.J. Resink, Expression of adhesion molecule T-cadherin is increased during neointima formation in experimental restenosis, Histochemistry and cell biology 118(4) (2002) 281-90.

[26] A. Frismantiene, D. Pfaff, A. Frachet, M. Coen, M.B. Joshi, K. Maslova, M.L. BochatonPiallat, P. Erne, T.J. Resink, M. Philippova, Regulation of contractile signaling and matrix 
1 remodeling by T-cadherin in vascular smooth muscle cells: constitutive and insulin2 dependent effects, Cellular signalling 26(9) (2014) 1897-908.

3 [27] Y.S. Kuzmenko, D. Stambolsky, F. Kern, V.N. Bochkov, V.A. Tkachuk, T.J. Resink, 4 Characteristics of smooth muscle cell lipoprotein binding proteins (p105/p130) as T-cadherin and regulation by positive and negative growth regulators, Biochemical and biophysical research communications 246(2) (1998) 489-94. [28] A. Frismantiene, B. Dasen, D. Pfaff, P. Erne, T.J. Resink, M. Philippova, T-cadherin promotes vascular smooth muscle cell dedifferentiation via a GSK3beta-inactivation dependent mechanism, Cellular signalling 28(5) (2016) 516-30. [29] T. Scott-Burden, T.J. Resink, U. Baur, M. Burgin, F.R. Buhler, Epidermal growth factor responsiveness in smooth muscle cells from hypertensive and normotensive rats, Hypertension 13(4) (1989) 295-304.

[32] T.Y. Li, S.Y. Lin, S.C. Lin, Mechanism and physiological significance of growth factorrelated autophagy, Physiology 28(6) (2013) 423-31.

[33] N. Mizushima, T. Yoshimori, B. Levine, Methods in mammalian autophagy research, Cell 140(3) (2010) 313-26.

[34] V.R. Solomon, H. Lee, Chloroquine and its analogs: a new promise of an old drug for effective and safe cancer therapies, European journal of pharmacology 625(1-3) (2009) 22033.

[35] Y.C. Kim, K.L. Guan, mTOR: a pharmacologic target for autophagy regulation, The Journal of clinical investigation 125(1) (2015) 25-32.

[36] S. Sridharan, K. Jain, A. Basu, Regulation of autophagy by kinases, Cancers 3(2) (2011) 2630-54.

[37] S.Y. Lin, T.Y. Li, Q. Liu, C. Zhang, X. Li, Y. Chen, S.M. Zhang, G. Lian, Q. Liu, K. Ruan, Z. Wang, C.S. Zhang, K.Y. Chien, J. Wu, Q. Li, J. Han, S.C. Lin, GSK3-TIP60-ULK1 signaling pathway links growth factor deprivation to autophagy, Science 336(6080) (2012) 477-81.

[38] R. Scherz-Shouval, Z. Elazar, Regulation of autophagy by ROS: physiology and pathology, Trends in biochemical sciences 36(1) (2011) 30-8.

[39] H.O. Rashid, R.K. Yadav, H.R. Kim, H.J. Chae, ER stress: Autophagy induction, inhibition and selection, Autophagy 11(11) (2015) 1956-1977.

[40] J.H. Kim, S.K. Hong, P.K. Wu, A.L. Richards, W.T. Jackson, J.I. Park, Raf/MEK/ERK can regulate cellular levels of LC3B and SQSTM1/p62 at expression levels, Experimental cell research 327(2) (2014) 340-52.

[41] G. Jia, G. Cheng, D.M. Gangahar, D.K. Agrawal, Insulin-like growth factor-1 and TNFalpha regulate autophagy through c-jun $\mathrm{N}$-terminal kinase and Akt pathways in human atherosclerotic vascular smooth cells, Immunology and cell biology 84(5) (2006) 448-54. 
1 [42] K.Y. Yu, Y.P. Wang, L.H. Wang, Y. Jian, X.D. Zhao, J.W. Chen, K. Murao, W. Zhu, L. 2 Dong, G.Q. Wang, G.X. Zhang, Mitochondrial KATP channel involvement in angiotensin IIinduced autophagy in vascular smooth muscle cells, Basic research in cardiology 109(4) 4 (2014) 416.

5 [43] J.C. Ibe, Q. Zhou, T. Chen, H. Tang, J.X. Yuan, J.U. Raj, G. Zhou, Adenosine monophosphate-activated protein kinase is required for pulmonary artery smooth muscle cell survival and the development of hypoxic pulmonary hypertension, American journal of respiratory cell and molecular biology 49(4) (2013) 609-18. [44] H. Zhang, Y. Gong, Z. Wang, L. Jiang, R. Chen, X. Fan, H. Zhu, L. Han, X. Li, J. Xiao, X. Kong, Apelin inhibits the proliferation and migration of rat PASMCs via the activation of $\mathrm{PI3K} / \mathrm{Akt} / \mathrm{mTOR}$ signal and the inhibition of autophagy under hypoxia, Journal of cellular and molecular medicine 18(3) (2014) 542-53.

[45] P. Haberzettl, B.G. Hill, Oxidized lipids activate autophagy in a JNK-dependent manner by stimulating the endoplasmic reticulum stress response, Redox biology 1 (2013) 56-64.

[46] B.G. Hill, P. Haberzettl, Y. Ahmed, S. Srivastava, A. Bhatnagar, Unsaturated lipid peroxidation-derived aldehydes activate autophagy in vascular smooth-muscle cells, The Biochemical journal 410(3) (2008) 525-34.

[47] C. He, H. Zhu, W. Zhang, I. Okon, Q. Wang, H. Li, Y.Z. Le, Z. Xie, 7-Ketocholesterol induces autophagy in vascular smooth muscle cells through Nox4 and Atg4B, The American journal of pathology 183(2) (2013) 626-37.

[48] K. Xu, Y. Yang, M. Yan, J. Zhan, X. Fu, X. Zheng, Autophagy plays a protective role in free cholesterol overload-induced death of smooth muscle cells, Journal of lipid research 51(9) (2010) 2581-90.

[49] J.K. Salabei, T.D. Cummins, M. Singh, S.P. Jones, A. Bhatnagar, B.G. Hill, PDGFmediated autophagy regulates vascular smooth muscle cell phenotype and resistance to oxidative stress, The Biochemical journal 451(3) (2013) 375-88.

[50] P. Hu, D. Lai, P. Lu, J. Gao, H. He, ERK and Akt signaling pathways are involved in advanced glycation end product-induced autophagy in rat vascular smooth muscle cells, International journal of molecular medicine 29(4) (2012) 613-8.

[51] H. Li, J. Li, Y. Li, P. Singh, L. Cao, L.J. Xu, D. Li, Y. Wang, Z. Xie, Y. Gui, X.L. Zheng, Sonic hedgehog promotes autophagy of vascular smooth muscle cells, American journal of physiology. Heart and circulatory physiology 303(11) (2012) H1319-31.

[52] M.O. Grootaert, P.A. da Costa Martins, N. Bitsch, I. Pintelon, G.R. De Meyer, W. Martinet, D.M. Schrijvers, Defective autophagy in vascular smooth muscle cells accelerates senescence and promotes neointima formation and atherogenesis, Autophagy 11(11) (2015) 2014-2032. 
1 [53] M.B. Joshi, M. Philippova, D. Ivanov, R. Allenspach, P. Erne, T.J. Resink, T-cadherin protects endothelial cells from oxidative stress-induced apoptosis, FASEB journal : official publication of the Federation of American Societies for Experimental Biology 19(12) (2005) $4 \quad 1737-9$.

[54] M. Philippova, M.B. Joshi, D. Pfaff, E. Kyriakakis, K. Maslova, P. Erne, T.J. Resink, Tcadherin attenuates insulin-dependent signalling, eNOS activation, and angiogenesis in vascular endothelial cells, Cardiovasc Res 93(3) (2012) 498-507. [55] B. Nyfeler, P. Bergman, E. Triantafellow, C.J. Wilson, Y. Zhu, B. Radetich, P.M. Finan, D.J. Klionsky, L.O. Murphy, Relieving autophagy and 4EBP1 from rapamycin resistance, Molecular and cellular biology 31(14) (2011) 2867-76.

[56] Z.H. Chen, J.F. Cao, J.S. Zhou, H. Liu, L.Q. Che, K. Mizumura, W. Li, A.M. Choi, H.H. Shen, Interaction of caveolin-1 with ATG12-ATG5 system suppresses autophagy in lung epithelial cells, American journal of physiology. Lung cellular and molecular physiology 306(11) (2014) L1016-25.

[57] D. Ivanov, M. Philippova, V. Tkachuk, P. Erne, T. Resink, Cell adhesion molecule Tcadherin regulates vascular cell adhesion, phenotype and motility, Experimental cell research 293(2) (2004) 207-18.

[58] E. Kyriakakis, M. Philippova, M.B. Joshi, D. Pfaff, V. Bochkov, T. Afonyushkin, P. Erne, T.J. Resink, T-cadherin attenuates the PERK branch of the unfolded protein response and protects vascular endothelial cells from endoplasmic reticulum stress-induced apoptosis, Cellular signalling 22(9) (2010) 1308-16.

[59] Y. Tang, Z. Liao, L. Han, Upregulation of Autophagy by Angiotensin II Triggers Phenotypic Switching of Aortic Vascular Smooth Muscle Cells, Journal of Clinical and Experimental Cardiology 5(4) (2014) DOI:10.4172/2155-9880.1000308.

[60] H. Takeuchi, Y. Kondo, K. Fujiwara, T. Kanzawa, H. Aoki, G.B. Mills, S. Kondo, Synergistic augmentation of rapamycin-induced autophagy in malignant glioma cells by phosphatidylinositol 3-kinase/protein kinase B inhibitors, Cancer research 65(8) (2005) 333646.

[61] W. Li, Q. Li, L. Qin, R. Ali, Y. Qyang, M. Tassabehji, B.R. Pober, W.C. Sessa, F.J. Giordano, G. Tellides, Rapamycin inhibits smooth muscle cell proliferation and obstructive arteriopathy attributable to elastin deficiency, Arteriosclerosis, thrombosis, and vascular biology 33(5) (2013) 1028-35.

[62] S. Verheye, W. Martinet, M.M. Kockx, M.W. Knaapen, K. Salu, J.P. Timmermans, J.T. Ellis, D.L. Kilpatrick, G.R. De Meyer, Selective clearance of macrophages in atherosclerotic plaques by autophagy, Journal of the American College of Cardiology 49(6) (2007) 706-15. 
1 [63] M.P. Philippova, V.N. Bochkov, D.V. Stambolsky, V.A. Tkachuk, T.J. Resink, T-cadherin 2 and signal-transducing molecules co-localize in caveolin-rich membrane domains of vascular 3 smooth muscle cells, FEBS letters 429(2) (1998) 207-10.

4 [64] T. Garofalo, P. Matarrese, V. Manganelli, M. Marconi, A. Tinari, L. Gambardella, A. 5 Faggioni, R. Misasi, M. Sorice, W. Malorni, Evidence for the involvement of lipid rafts localized at the ER-mitochondria associated membranes in autophagosome formation, Autophagy 12(6) (2016) 917-35.

8 [65] iLIR Autophagy Database. <https://ilir.warwick.ac.uk/kwresult.php>).

9 [66] P. Wild, D.G. McEwan, I. Dikic, The LC3 interactome at a glance, Journal of cell science 10 127(Pt 1) (2014) 3-9.

11 [67] A.B. Birgisdottir, T. Lamark, T. Johansen, The LIR motif - crucial for selective autophagy, Journal of cell science 126(Pt 15) (2013) 3237-47.

[68] Y. Fujishima, N. Maeda, K. Matsuda, S. Masuda, T. Mori, S. Fukuda, R. Sekimoto, M. Yamaoka, Y. Obata, S. Kita, H. Nishizawa, T. Funahashi, B. Ranscht, I. Shimomura, Adiponectin association with T-cadherin protects against neointima proliferation and atherosclerosis, FASEB journal : official publication of the Federation of American Societies for Experimental Biology (2017).

[69] E. Kyriakakis, K. Maslova, M. Philippova, D. Pfaff, M.B. Joshi, S.A. Buechner, P. Erne, T.J. Resink, T-Cadherin is an auxiliary negative regulator of EGFR pathway activity in cutaneous squamous cell carcinoma: impact on cell motility, The Journal of investigative dermatology 132(9) (2012) 2275-85.

[70] Y. Adachi, T. Takeuchi, H. Sonobe, Y. Ohtsuki, An adiponectin receptor, T-cadherin, was selectively expressed in intratumoral capillary endothelial cells in hepatocellular carcinoma: possible cross talk between T-cadherin and FGF-2 pathways, Virchows Archiv : an international journal of pathology 448(3) (2006) 311-8.

[71] E. Kyriakakis, K. Maslova, A. Frachet, N. Ferri, A. Contini, D. Pfaff, P. Erne, T.J. Resink, M. Philippova, Cross-talk between EGFR and T-cadherin: EGFR activation promotes Tcadherin localization to intercellular contacts, Cellular signalling 25(5) (2013) 1044-53. 


\section{FIGURE LEGENDS}

Figure 1. T-cadherin upregulation in SMCs enhances autophagic flux under conditions of serum-deprivation. Subconfluent cultures of rat (R-SMC) and murine (M-SMC) aortic SMCs stably transduced with full length human T-cadherin cDNA (Tcad+) or empty vector (E) were serum-deprived for $6 \mathrm{~h}$ without (control conditions, ctrl) or with inclusion of chloroquine ( $C Q$, $30 \mu \mathrm{M})$. Thereafter whole cell lysates were collected for immunoblotting (A, B), or live cells were stained in-well for autophagic vacuole accumulation using Cyto-ID® $(C, D)$. (A, B) Representative immunoblots of whole cell lysates probed for T-cadherin, LC3, and for tubulin as internal loading control. Histograms present LC3-II abundancies expressed relative to the levels in E-SMCs under control conditions. (C, D) Representative fluorescence images of SMCs stained with Cyto-ID® (green) and Hoechst 33342 nuclear dye (blue). Scale bar: 50 $\mu \mathrm{m}$. Histograms present quantification of autophagosomes/cell. Data are shown as the mean \pm S.D. of three to five independent experiments. ${ }^{*} P<0.05 ;{ }^{* \star} P<0.01 ;{ }^{* *} P<0.001$.

Figure 2. T-cadherin upregulation in SMCs increases autophagic flux as assessed by staining of autophagosome markers LC3B and p62. Subconfluent cultures of rat Tcad+-SMC and control E-SMCs were serum-deprived for $6 \mathrm{~h}$ without (control condition) or with inclusion of chloroquine (CQ, $30 \mu \mathrm{M})$. After fixation and permeabilization, SMCs were stained with primary anti-LC3B (A) or anti-p62 (B) antibodies and secondary Alexa Fluor 488-conjugated IgGs for detection of autophagosomes (green). Nuclei were counterstained using Hoechst 33342 (blue). Representative fluorescence images of stained SMCs are shown. Scale bar: $50 \mu \mathrm{m}$. Data for quantification of autophagosomes/cell is given as the mean \pm S.D. of three independent experiments. ${ }^{*} P<0.05 ;{ }^{* *} P<0.01$; $\left.{ }^{* *} P<0.001\right)$.

Figure 3. T-cadherin-enhanced autophagy in SMCs is independent of Akt/mTORC1 and ROS. Subconfluent cultures of R-SMC (A-D) and M-SMC (E-H) E and Tcad+ transductants were serum-deprived $6 \mathrm{~h}$ without (control; ctrl) or with inclusion of either rapamycin (rapa, 250 nM; panels A, E), LY294002 (LY, $20 \mu \mathrm{M}$; panels B, F) or N-acetyl-L-cysteine (NAC, 20 mM; panels C, G). Whole cell lysates were variously immunoblotted for autophagy marker LC3-II, for PI3K/Akt/mTORC1 axis effectors to control for efficacy of mTOR and PI3K inhibition and for tubulin as internal loading control. Representative blots are shown. Histograms (panels D, H) present quantification of LC3-II abundancies expressed relative to abundancy in E-SMCs under control conditions. Data are shown as the mean \pm S.D. of three independent experiments. Significant differences between E- and Tcad+-SMCs are indicated with asterisks $\left({ }^{\star} P<0.05 ;{ }^{\star *} P<0.01 ;{ }^{* \star *} P<0.001\right)$.

Figure 4. T-cadherin-enhanced autophagy in SMCs is independent of UPR activation. Subconfluent cultures of R-SMC (A) and M-SMC (B) transductants were serum-deprived for 
$16 \mathrm{~h}$ without (control; ctrl) or with inclusion o tunicamycin (TUN, $3 \mu \mathrm{g} / \mathrm{ml}$ ). Whole cell lysates were variously immunoblotted for autophagy marker LC3-II and/or UPR activation markers Grp78 or p-elF2 $\alpha$. Representative blots are shown and histograms present marker abundancies expressed relative to the respective levels in E-SMCs under control conditions. Data are shown as the mean \pm S.D. of three to five independent experiments. ${ }^{*} P<0.05 ;{ }^{* \star} P$ $<0.01 ;{ }^{* *} P<0.001$.

Figure 5. T-cadherin-enhanced autophagic flux in SMCs is attenuated by MEK1/2/Erk1/2 inhibition. Subconfluent cultures of R-SMC $(A, C)$ and M-SMC (B, D) transductants were serum-deprived for 6h without (control; ctrl) or with inclusion of MEK1/2 inhibitor PD98059 $(P D, 10 \mu \mathrm{M})$. Whole cell lysates were collected for immunoblotting $(A, B)$ or in-well staining for autophagic vacuole accumulation was performed (C, D). (A, B) Representative immunoblots of whole cell lysates probed for LC3 and $p$-Erk1/2, and for tubulin as internal loading control. Histograms present LC3-II and p-Erk1/2 abundancies expressed relative to the respective levels in E-SMCs under control conditions. (C, D) Representative fluorescence images of SMCs stained with Cyto-ID® (green) and Hoechst 33342 nuclear dye (blue). Scale bar: $50 \mu \mathrm{m}$. Histograms present quantification of autophagosomes/cell expressed relative to that in E-SMCs under control conditions. Data are shown as the mean \pm S.D. of three to four independent experiments. ${ }^{*} P<0.05 ;{ }^{* *} P<0.01 ;{ }^{* *} P<0.001$.

Figure 6. SMC survival under conditions of serum-deprivation is augmented by upregulation of T-cadherin and is sensitive to MEK1/2/Erk1/2 or autophagy inhibition. Subconfluent cultures of R-SMC (A, C) and M-SMC (B) transductants were cultured for 3 days under conditions of serum-deprivation without (control; ctrl) or with inclusion of either MEK $1 / 2$ inhibitor PD98059 (PD, $10 \mu \mathrm{M})$, autophagy inhibitor chloroquine (CQ, $30 \mu \mathrm{M})$ or UO126 (UO, $5 \mu \mathrm{M})$. Survival was evaluated by enumeration of adherent cells on days 1, 2 and 3 expressed relative to the starting cell number on day 0 (arbitrarily taken as $100 \%$ ). Data are shown as the mean \pm S.E.M. of four independent experiments. $P$ values for the given pairs were obtained by nonlinear regression analysis.

Figure 7. Autophagic flux induced by serum-deprivation is attenuated in T-cadherin deficient SMCs. Subconfluent cultures of SMCs isolated from wildtype $\left(\mathrm{Cdh} 13^{+/+}\right)$and T-cadherin knockout $\left(\mathrm{Cdh}_{13^{--}}\right)$mice $(\mathrm{A}, \mathrm{C})$, or rat aortic SMCs transduced with rat T-cadherin shRNA (shTcad) or non-target shRNA as control (shC) (B, D) were serum-deprived for $6 \mathrm{~h}$ without (control conditions) or with inclusion of chloroquine $(C Q, 30 \mu M)$. (A, B) Whole cell lysates were collected for immunoblotting. Representative immunoblots of whole cell lysates probed for T-cadherin, LC3B, p-Erk1/2 (and tubulin as internal loading control). LC3-II and p-Erk1/2 abundancies are expressed relative to respective levels in the Cdh $13^{+/+}-$SMCs or shC-SMCs 
1 under control conditions. (C, D) Cells were stained for autophagosome accumulation using

2 primary anti-p62/secondary Alexa Fluor 488-conjugated IgGs. Representative fluorescence

3 images of SMCs stained for p62 (green) and Hoechst 33342 nuclear dye (blue) are shown.

4 Scale bar: $50 \mu \mathrm{m}$. Quantification of autophagosomes/cell is given as mean \pm S.D. of three

5 independent experiments. ${ }^{*} P<0.05 ;{ }^{* *} P<0.01$; ${ }^{* * *} P<0.001$.

6 Figure 8. T-cadherin deficient SMCs exhibit a survival disadvantage under conditions of

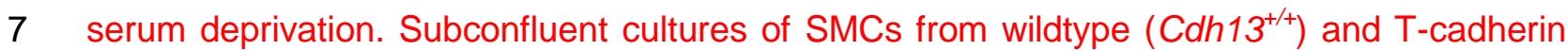
8 knockout $\left(\mathrm{Cdh}_{13^{-}}\right)$mice were serum-deprived for 3 days. (A) Survival was evaluated by 9 enumeration of adherent cells on days 1,2 and 3 expressed relative to the starting cell 10 number on day 0 (arbitrarily taken as $100 \%$ ). Data are shown as the mean \pm S.E.M. of three 11 independent experiments. $P$ value was obtained by nonlinear regression analysis. (B) Cell 12 apoptosis was analysed on day 3 by FITC-Annexin V/propidium iodide (PI) dual color flow 13 cytometry. Representative compensated flow cytometric dot-plots are shown. Viable cells 14 were annexin $\mathrm{V}$ and $\mathrm{PI}$ negative (lower left (LL) quadrant); dead cells were PI positive and annexin $\mathrm{V}$ negative (upper left (UL) quadrant); early apoptotic cells were annexin $\mathrm{V}$ positive and $\mathrm{PI}$ negative (lower right (LR) quadrant); late apoptotic cells were annexin $\mathrm{V}$ and $\mathrm{PI}$ positive (upper right (UR) quadrant). The percentages of early and late apoptotic cell populations were calculated and shown in the histogram. Data are mean \pm S.D of three independent experiments. ${ }^{* *} P<0.01 ;{ }^{* *} P<0.001$.

Figure 9. Schematic diagram illustrating the mechanism whereby T-cadherin augments autophagy-dependent survival of SMCs under conditions of serum-deprivation. Signaling pathways affected positively in SMCs with upregulated T-cadherin expression are depicted (+). T-cadherin upregulation augments autophagy during serum-starvation in a manner that is dependent on MEK1/2/Erk1/2 signaling cascade (solid green lines), and is independent of the PI3K/Akt/mTORC1 signaling pathway (dashed black lines). Molecular partners and immediate downstream effectors enabling cell membrane-located GPI-anchored T-cadherin to modify activity of intracellular stress signaling pathways are unknown (indicated with ?). 
Figure 1

A

$R$-SMC

T-cadherin

$$
\frac{\text { ctrl }}{\mathrm{E} \text { Tcad }+} \frac{+\mathrm{CQ}}{\mathrm{E} \text { Tcad }+}
$$

LC3-II-

tubulin

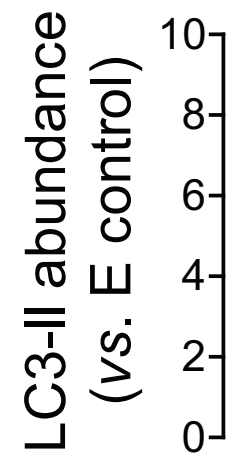

*

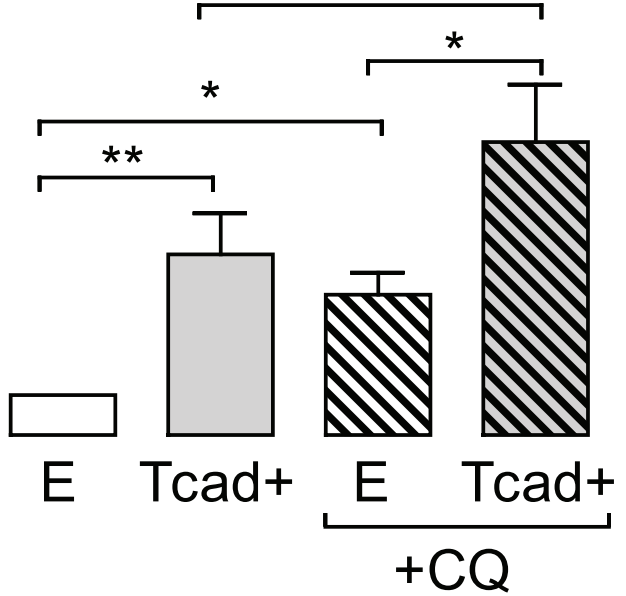

C

\section{ctrl $\mathrm{R}-\mathrm{SMC}+\mathrm{CQ}$}
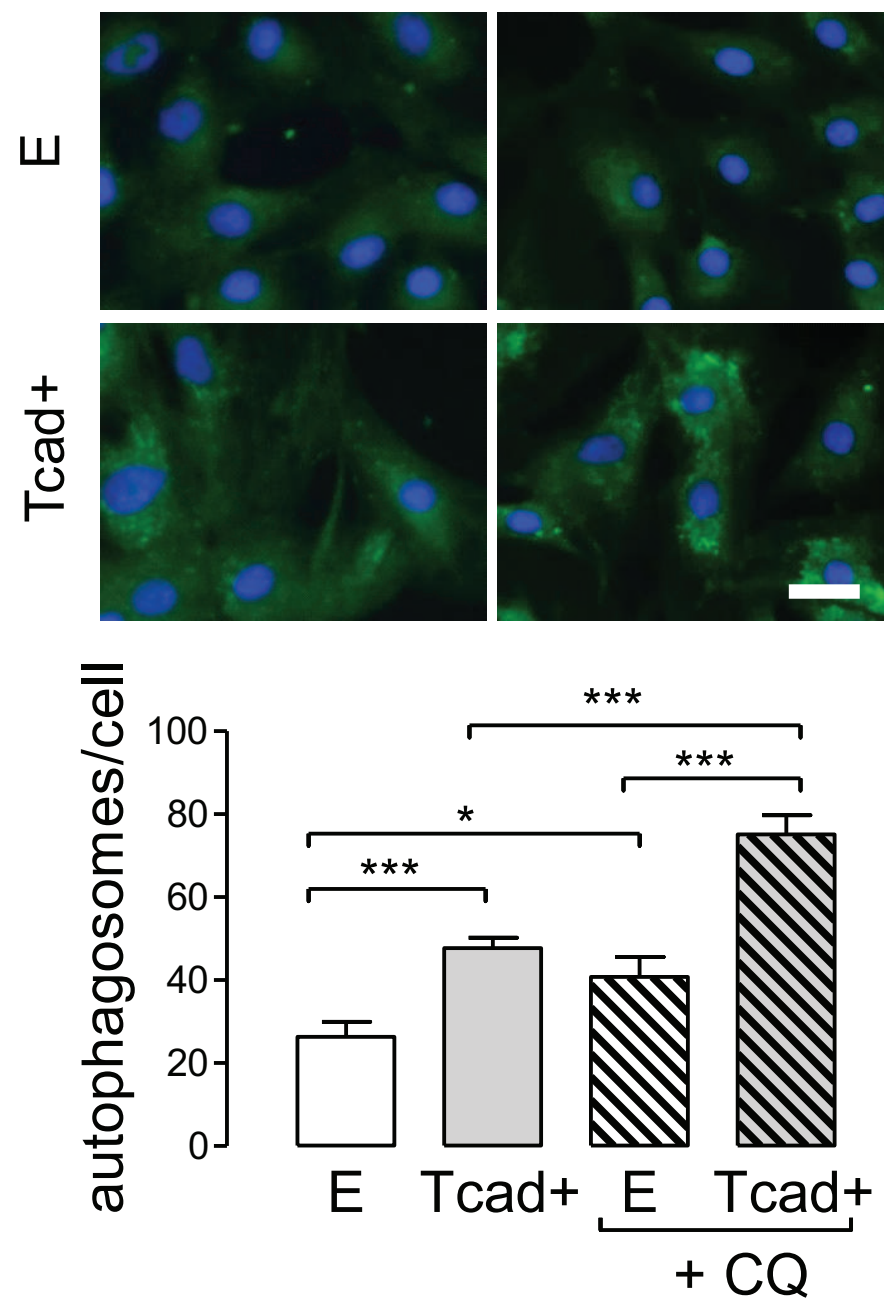

B

$\mathrm{kDa}$

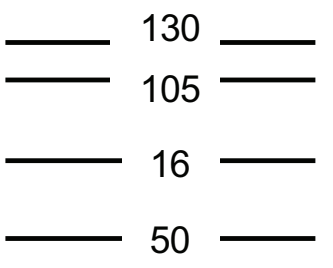

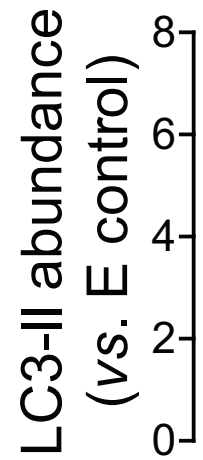

D
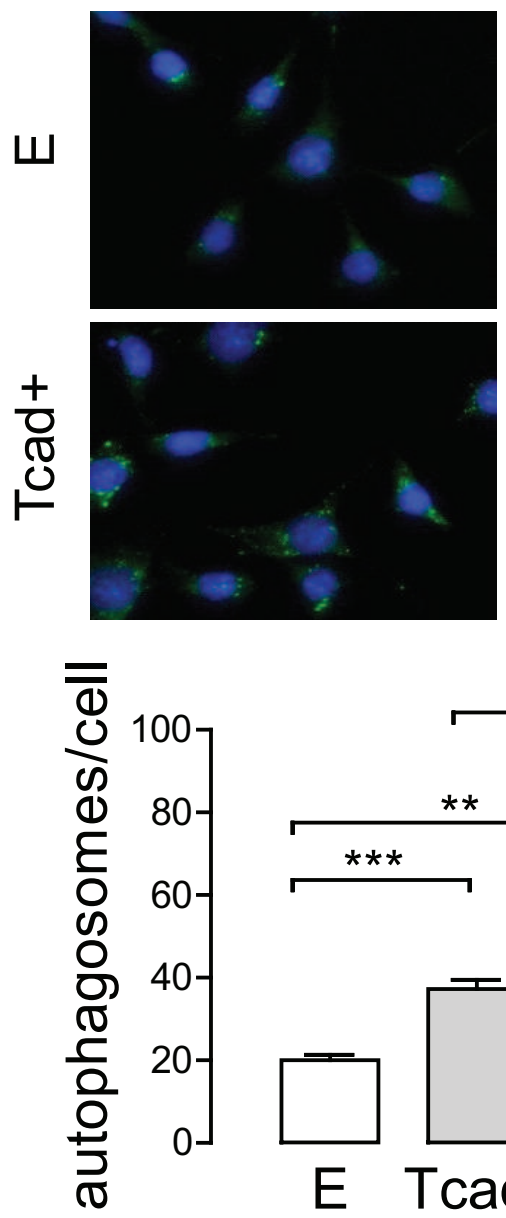
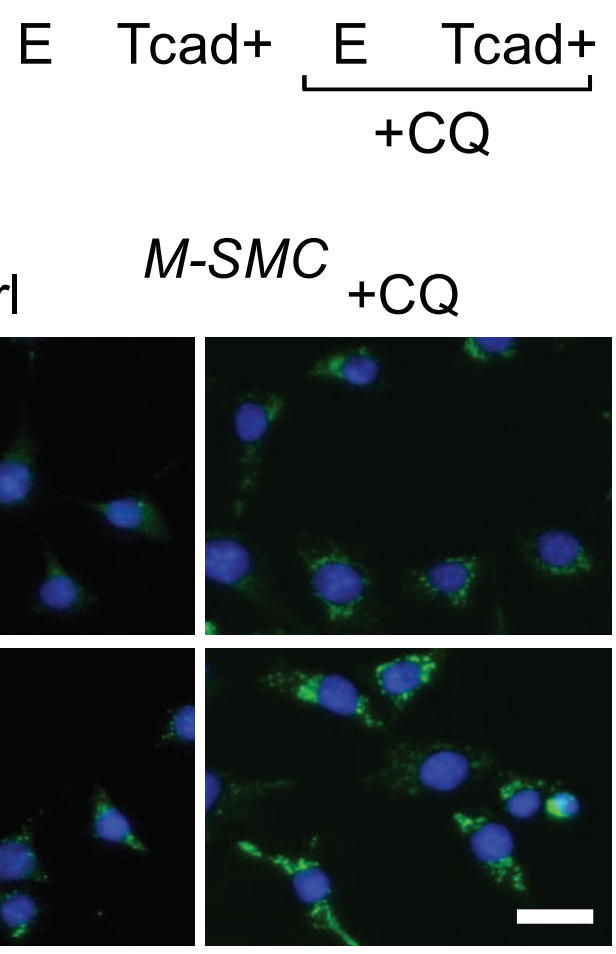

ctrl $M-S M C+C Q$

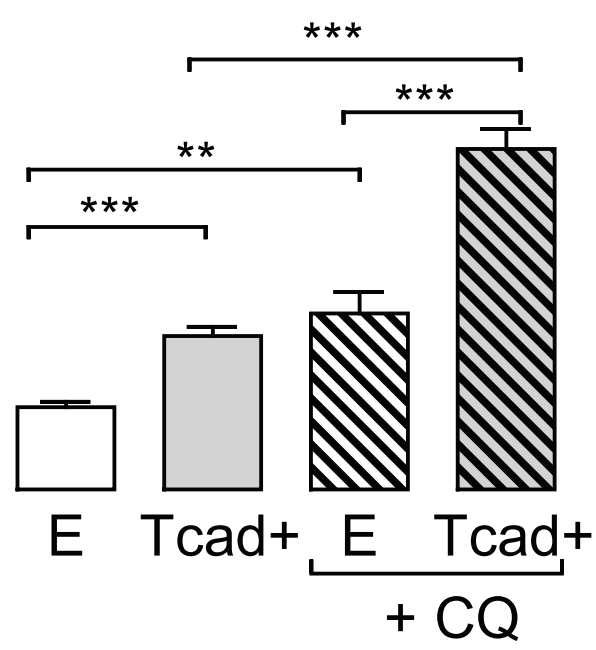

M-SMC

\begin{tabular}{|c|c|}
\hline ctrl & $+C Q$ \\
\hline E Tcad+ & Tcad+ \\
\hline
\end{tabular}

T-cadherin - LC3-II tubulin 
Figure 2

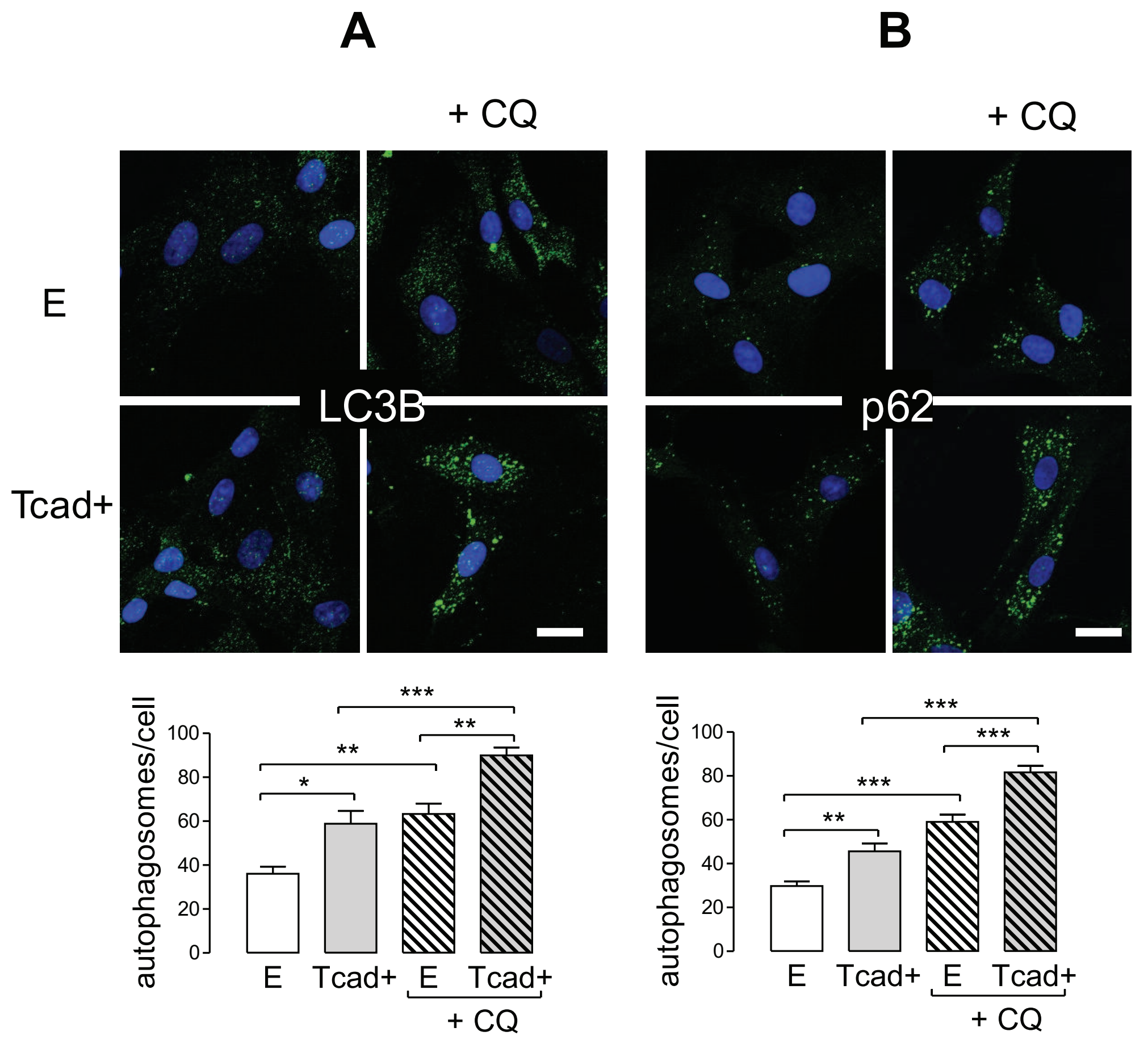


Figure 3
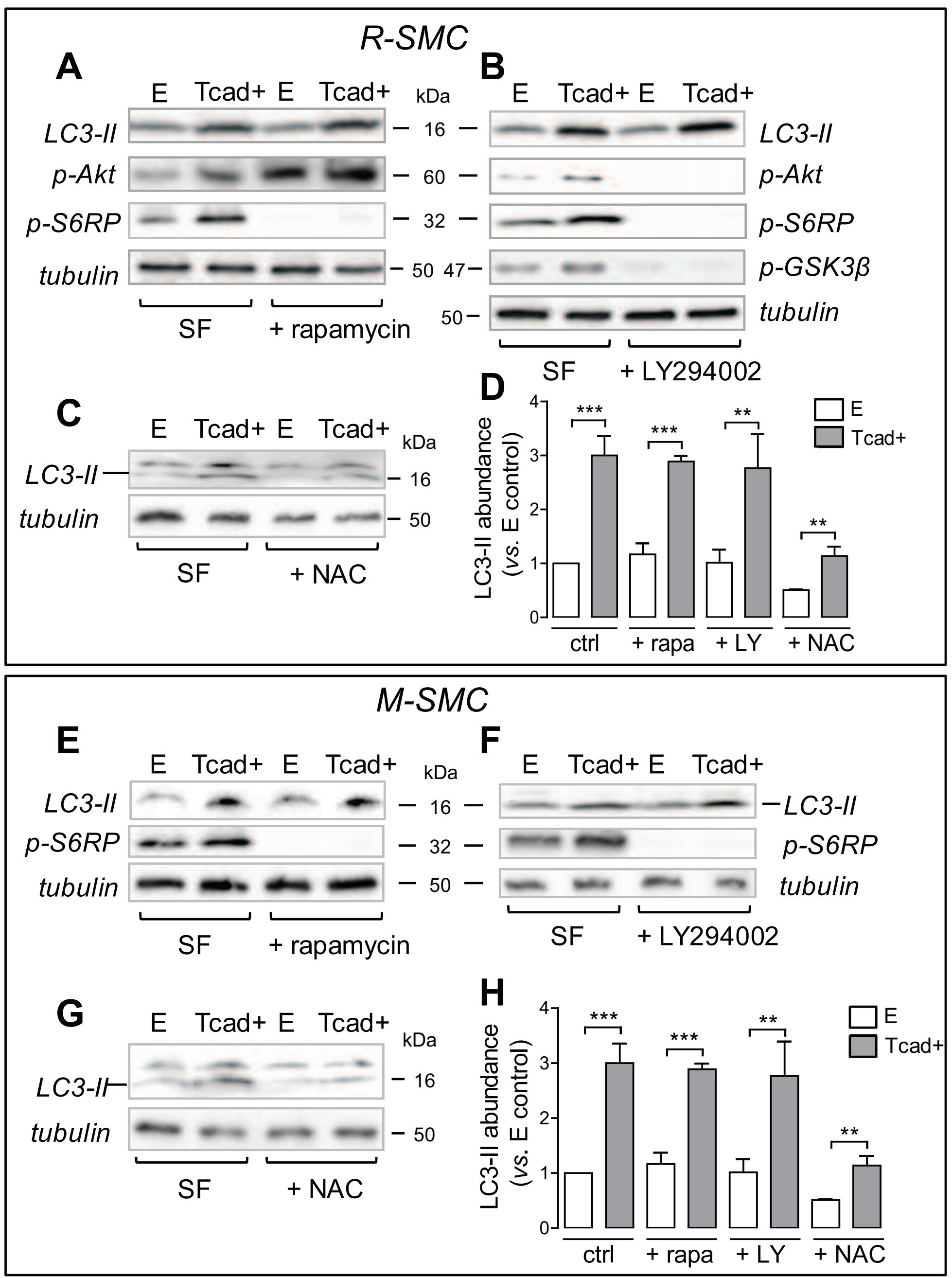
Figure 4

R-SMC
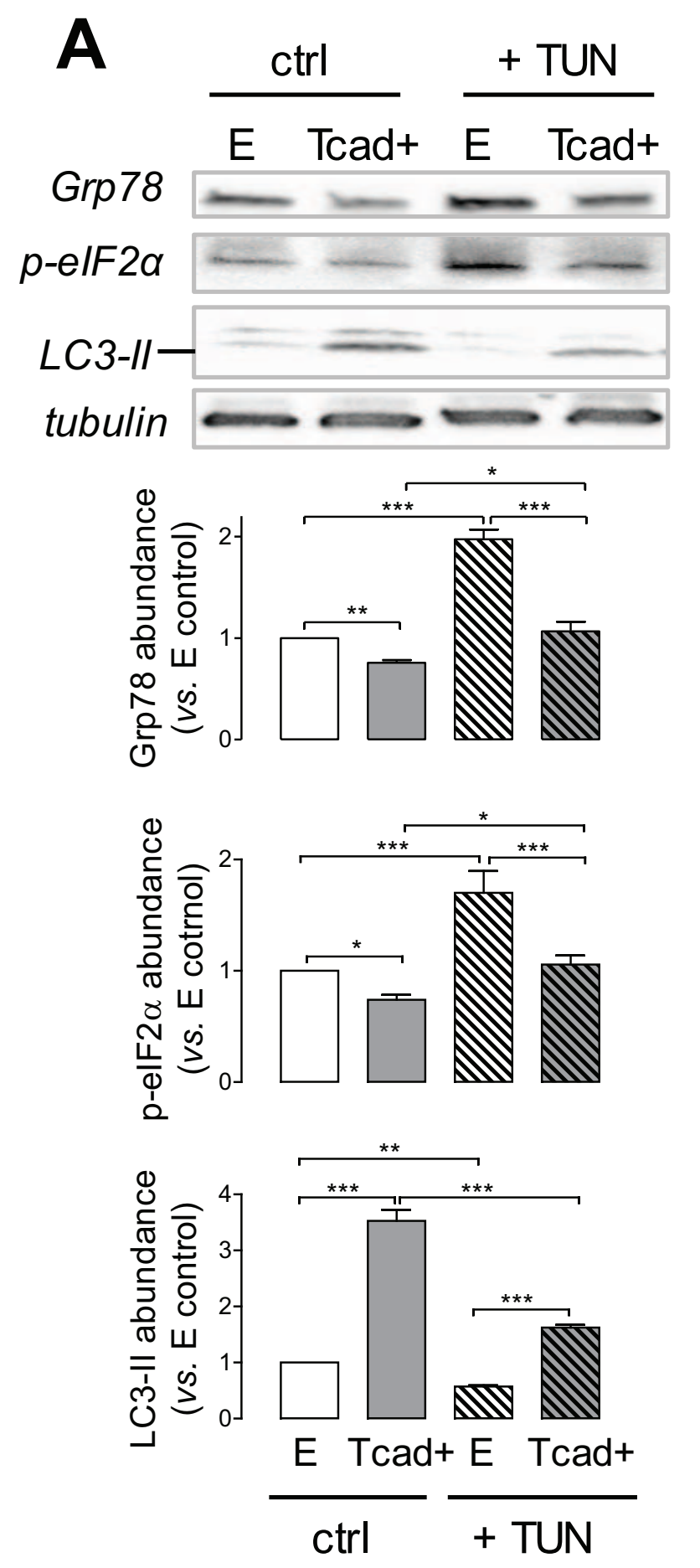

M-SMC

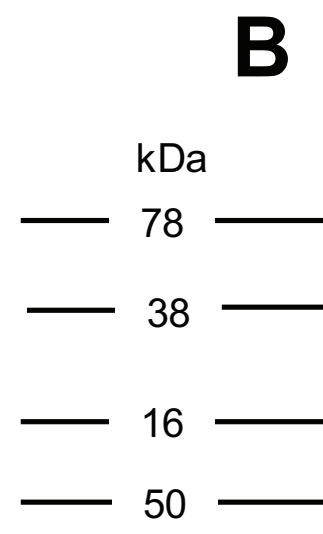

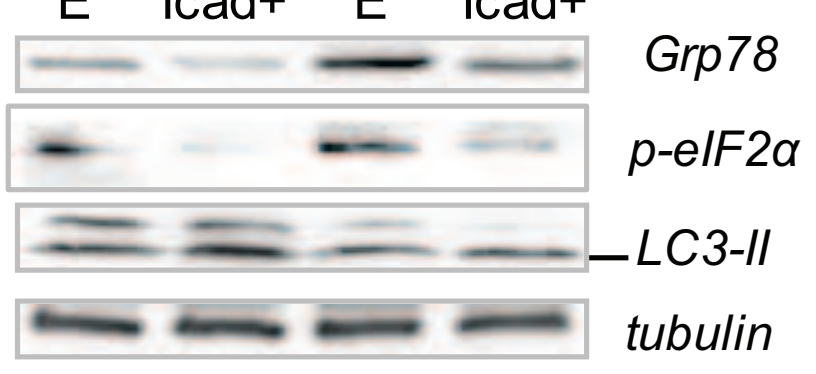
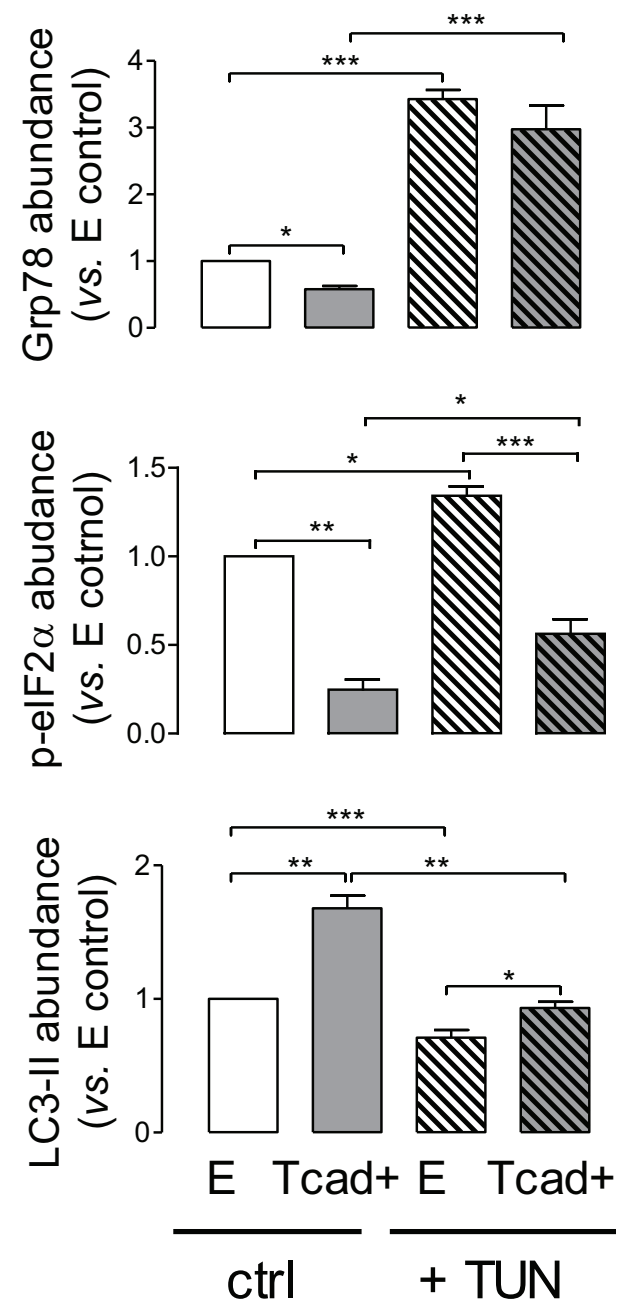
Figure 5

M-SMC

A

\section{R-SMC \\ $\frac{\text { ctrl }}{\text { E Tcad+ }} \frac{+ \text { PD }}{\text { E Tcad+ }}$}

LC3-II - - - - - - -

$p-E R K 1 / 2=-$

tubulin
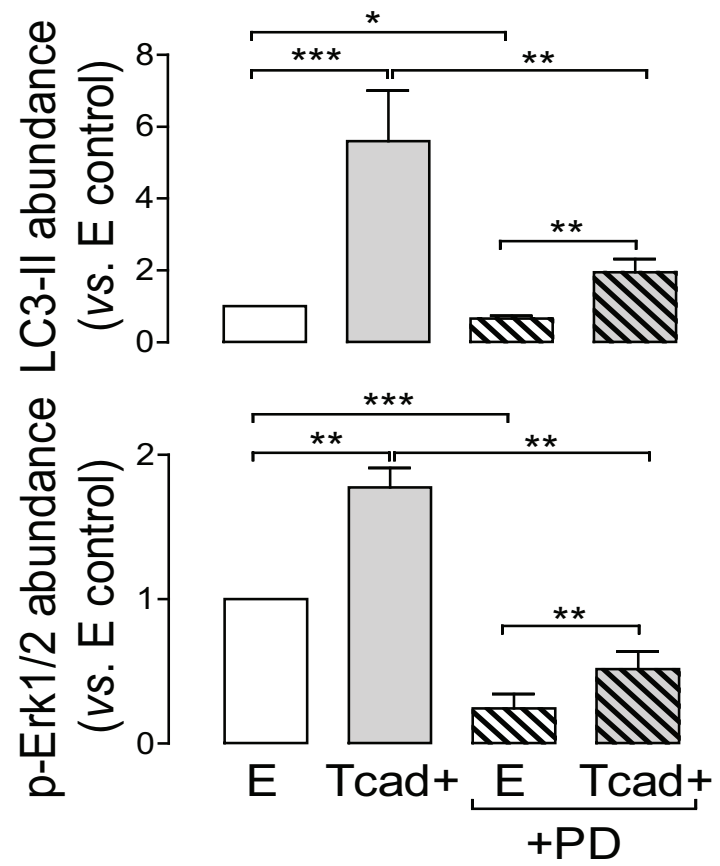

C
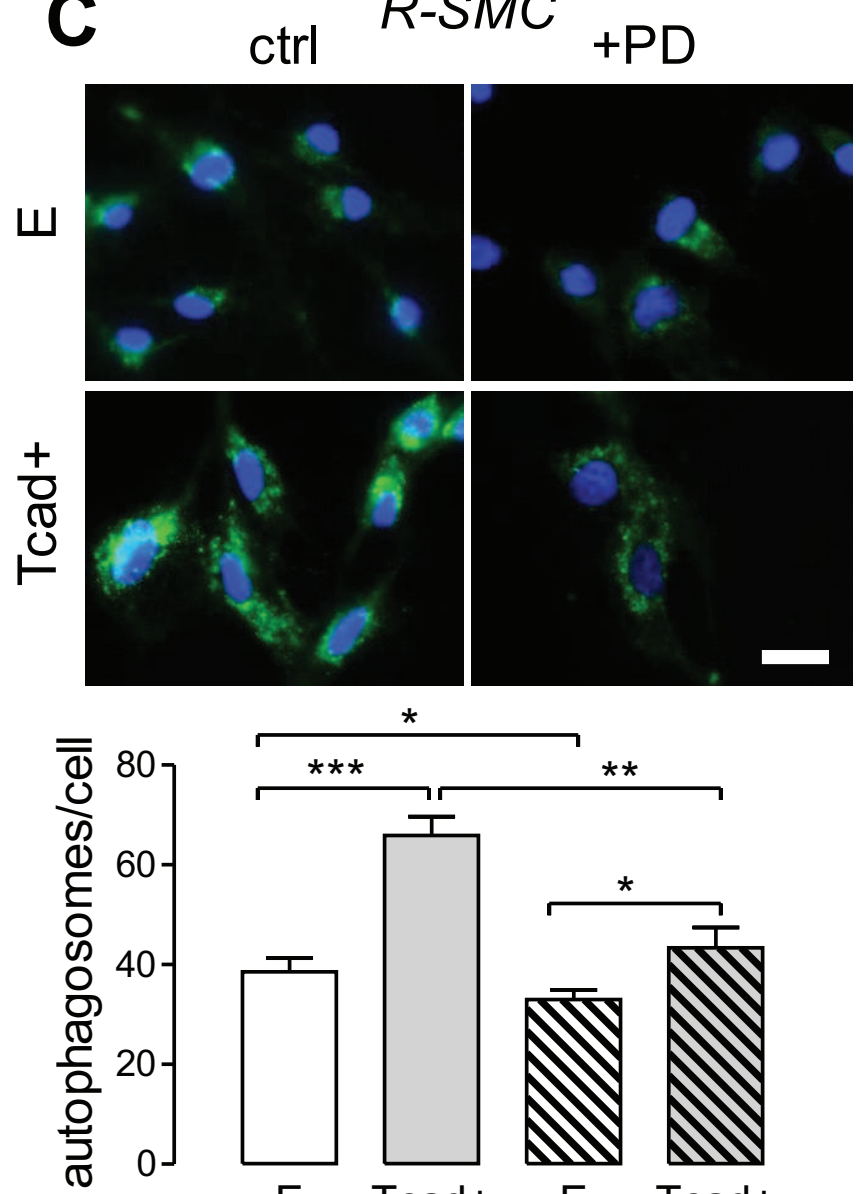

*

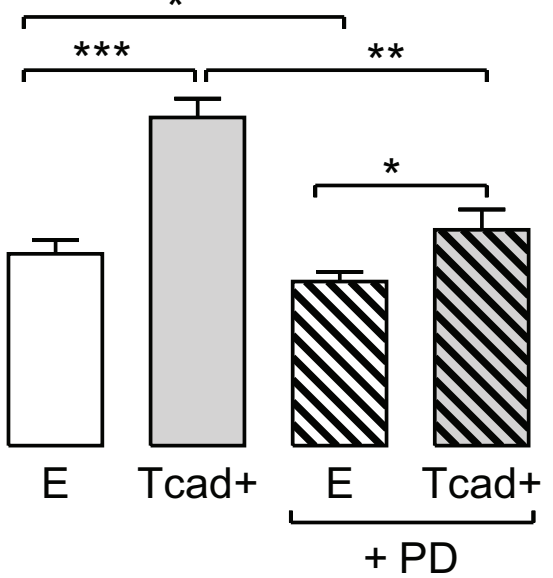

$\mathrm{kDa}$

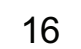

$44 / 42$

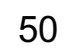

B $\frac{\text { ctrl }}{E_{\text {Tcad }+}} \frac{+ \text { PD }}{\text { E Tcad+ }}$

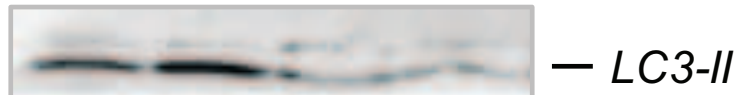
$p-E R K 1 / 2$ tubulin
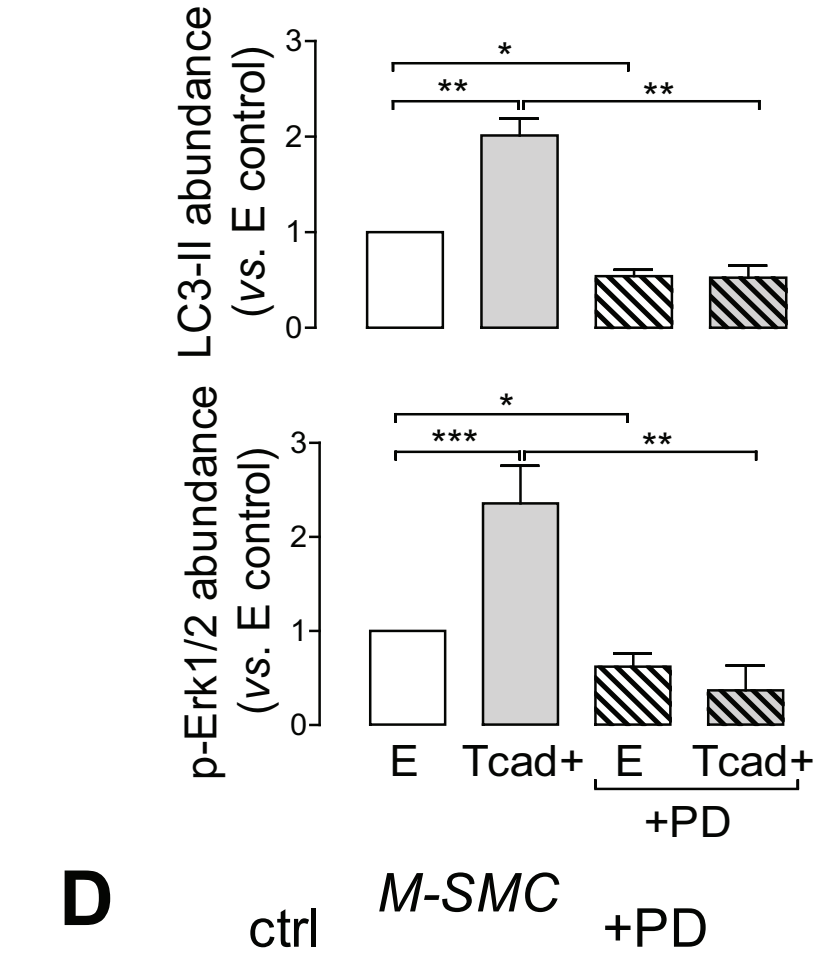
ctrl
M-SMC
$+P D$
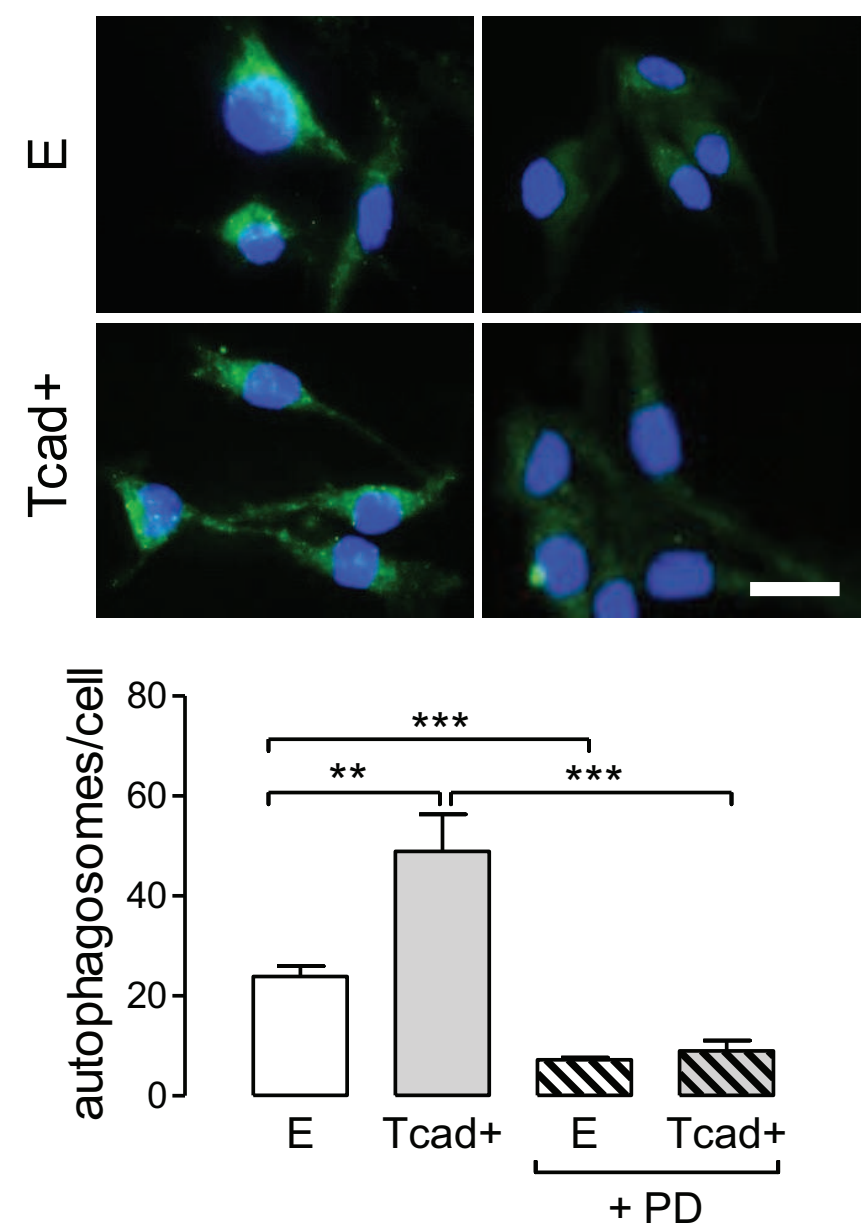
Figure 6
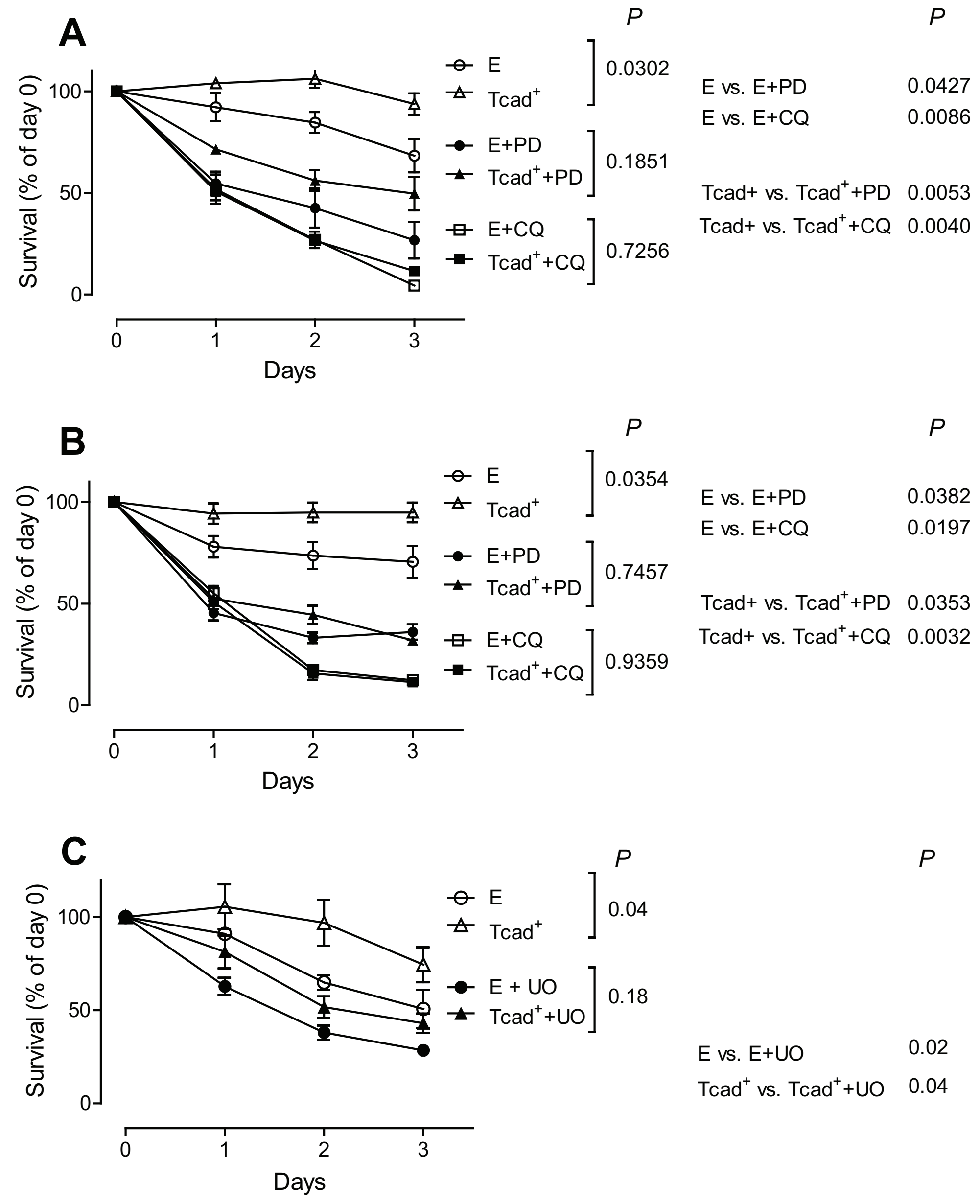
Figure 7

A

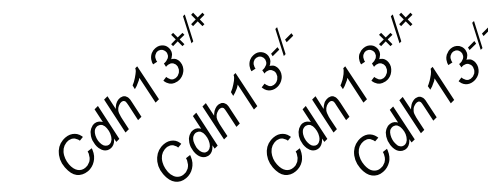

B

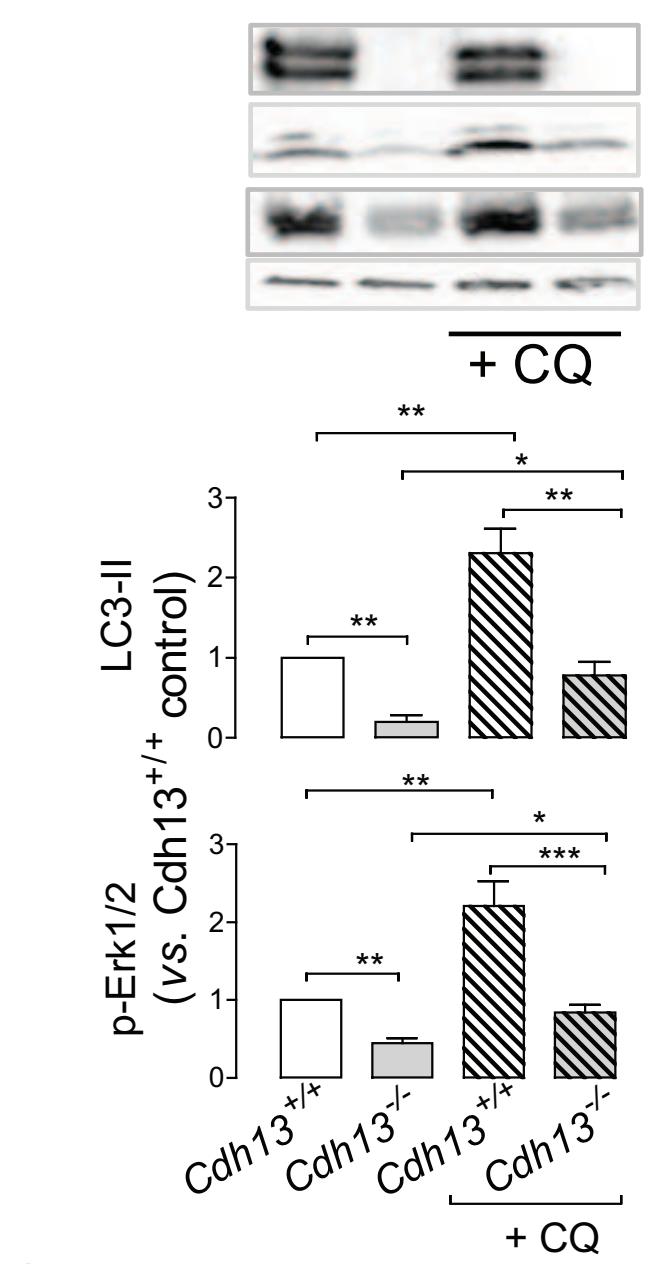

T-cadherin

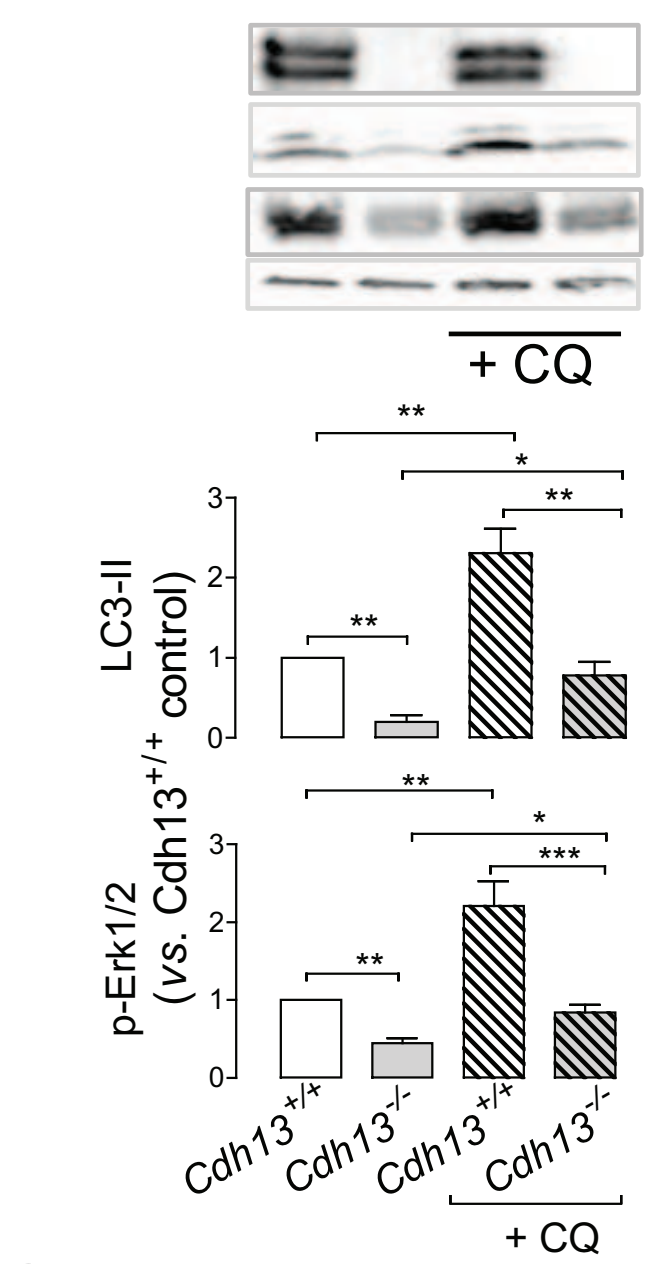

LC3-II p-ERK $1 / 2$ tubulin

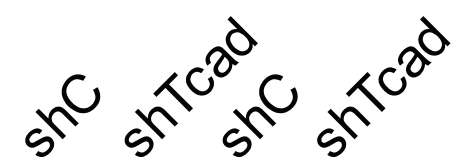

C
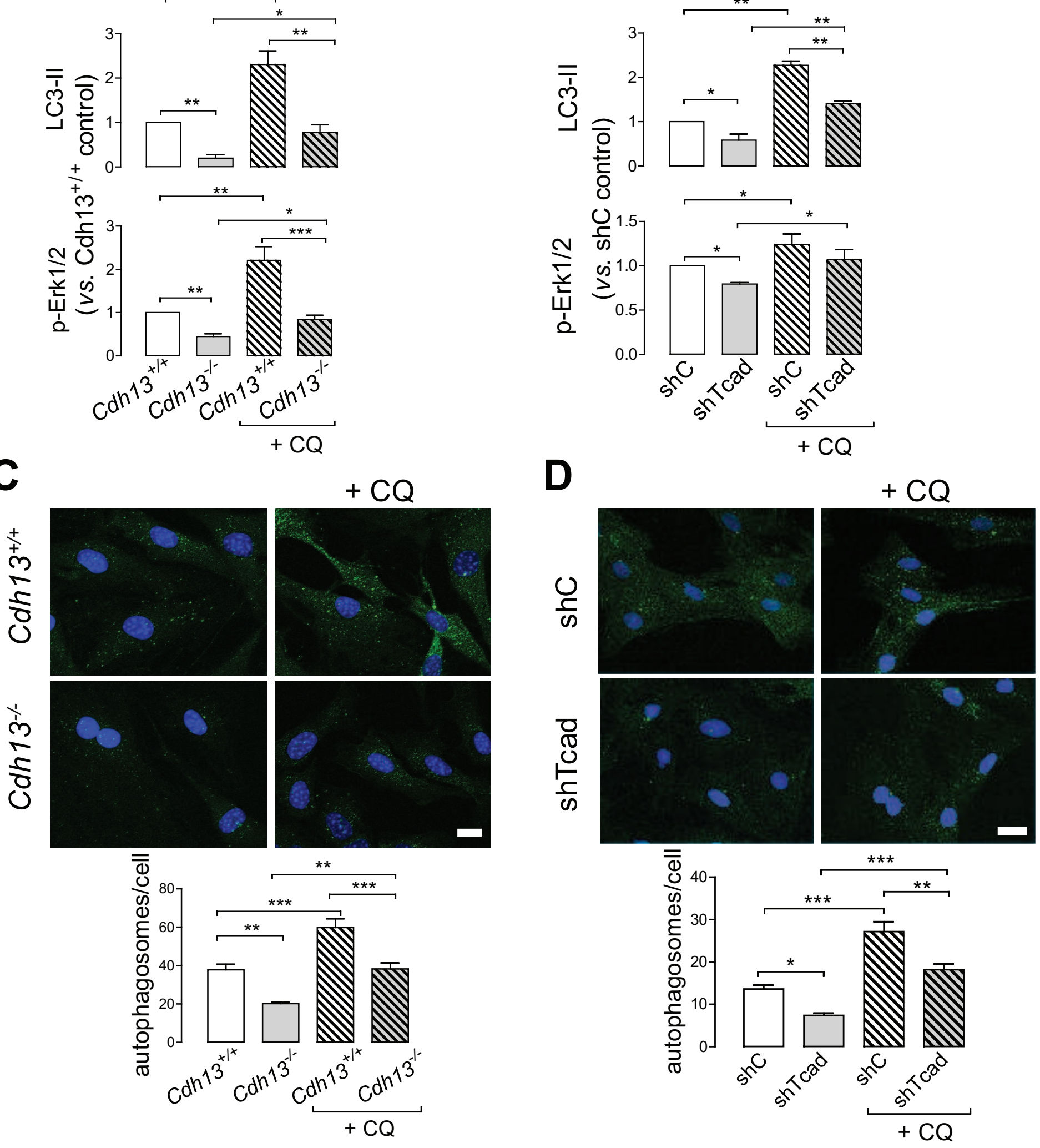
Figure 8

A

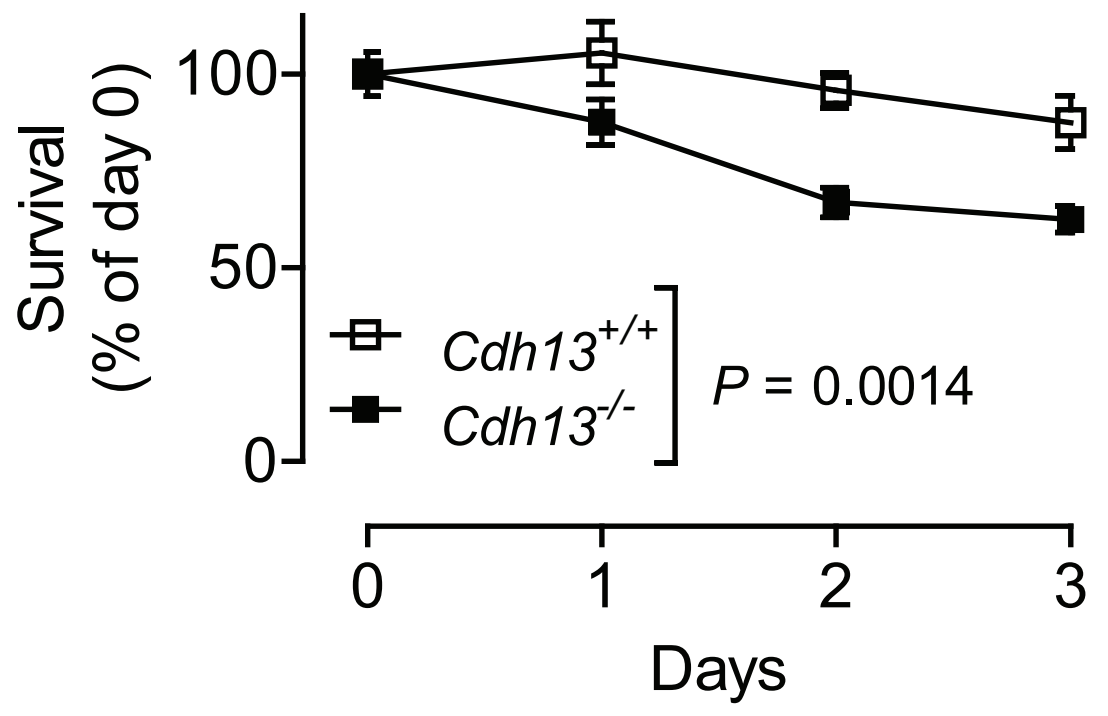

B
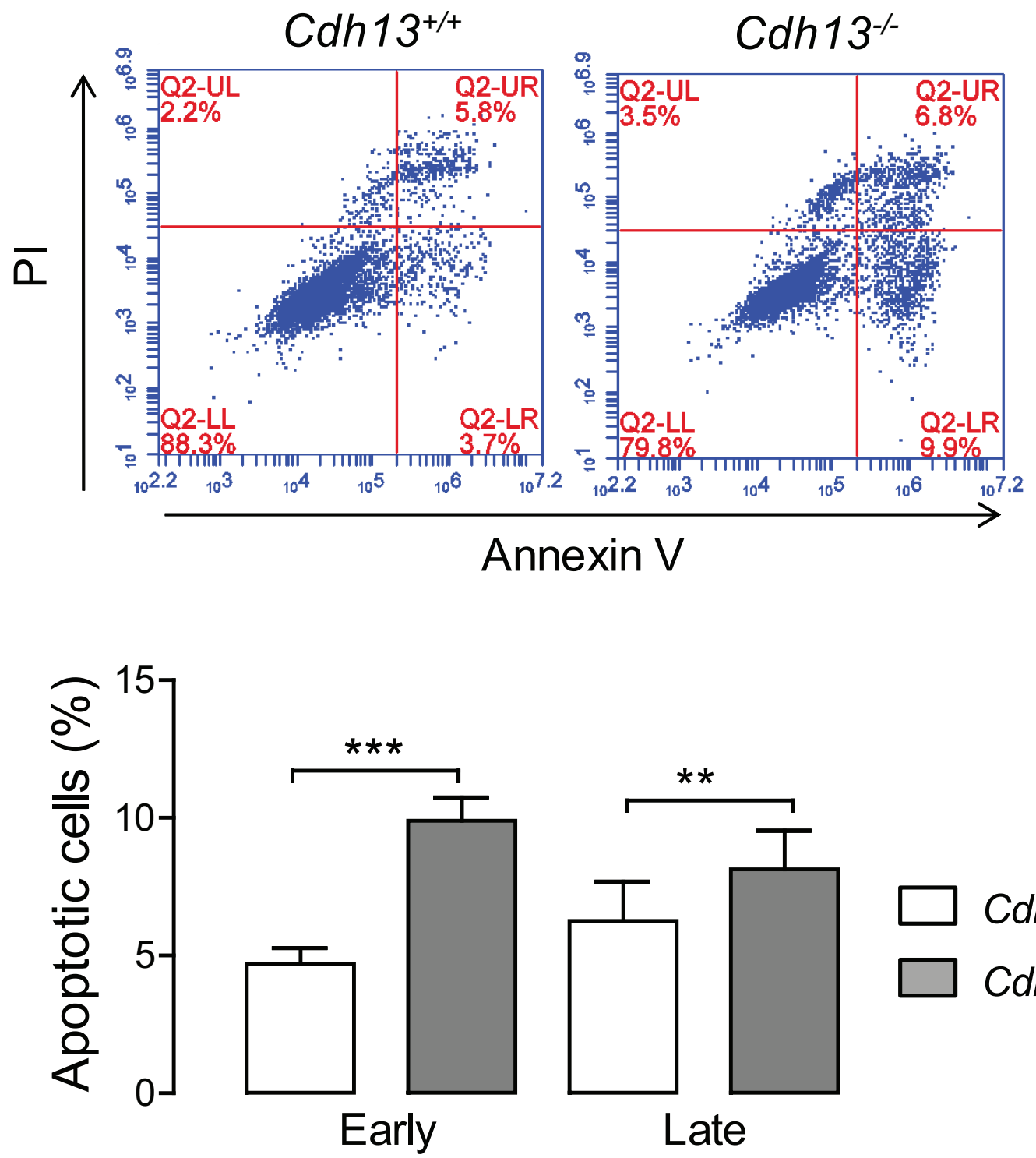

$\square \mathrm{Cdh} 13^{+/+}$

$\square$ Cdh13--

$\left(\right.$ Annexin $\left.\mathrm{V}^{+} / \mathrm{PI}^{-}\right) \quad\left(\right.$ Annexin $\left.\mathrm{V}^{+} / \mathrm{PI}^{+}\right)$ 


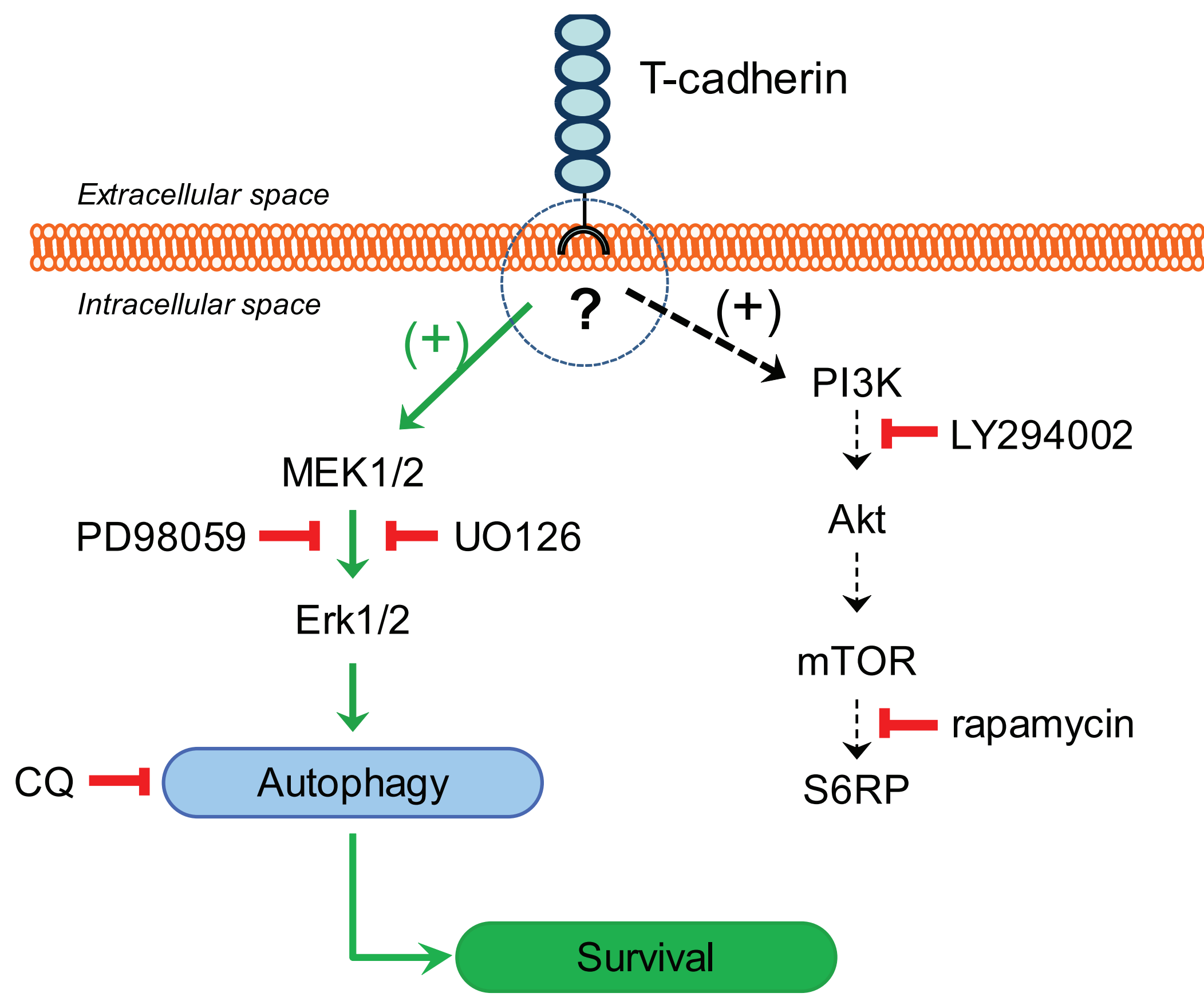




\section{T-cadherin}

Extracellular space

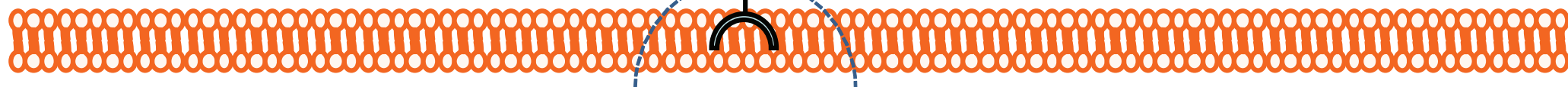
Intracellular space

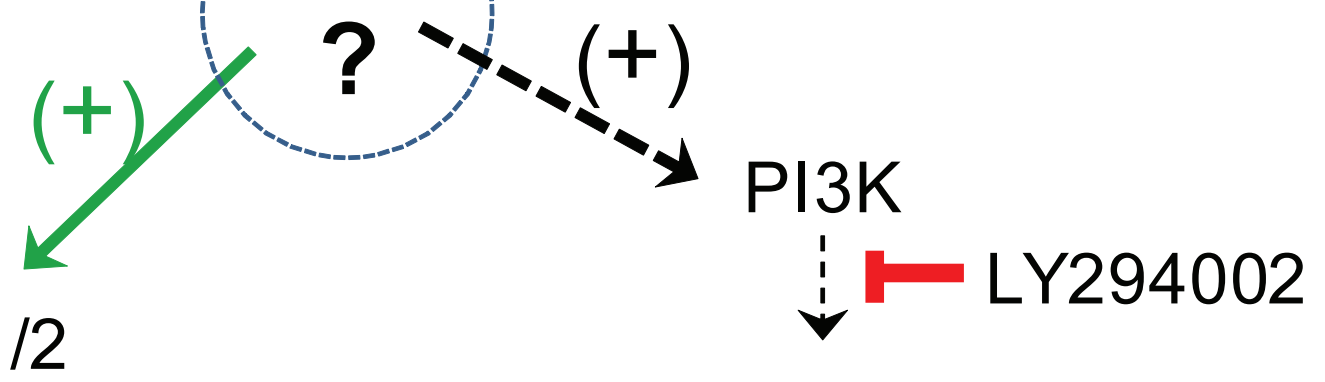

MEK1/2

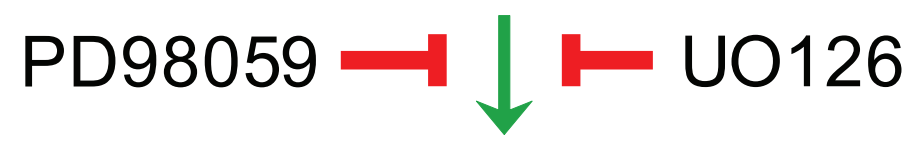

Erk1/2

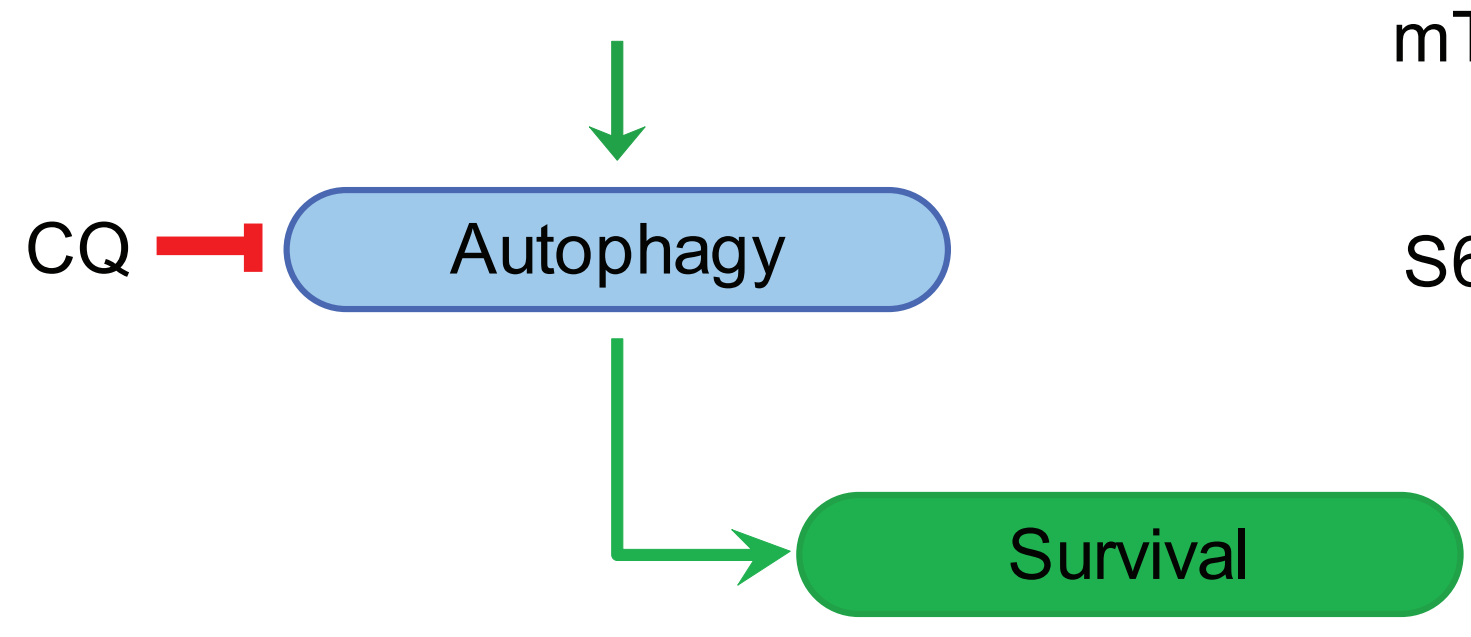

Akt

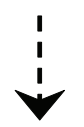

mTOR

rapamycin S6RP 